\title{
Horizons of semiclassical black holes are cold
}

\author{
Ram Brustein ${ }^{a, b}$ and A.J.M. Medved ${ }^{c}$ \\ ${ }^{a}$ Department of Physics, Ben-Gurion University, \\ Beer-Sheva 84105, Israel \\ ${ }^{b}$ CAS, Ludwig-Maximilians-Universität München, \\ 80333 München, Germany \\ ${ }^{c}$ Department of Physics $\&$ Electronics, Rhodes University, \\ Grahamstown 6140, South Africa \\ E-mail: ramyb@bgu.ac.il, j.medved@ru.ac.za
}

ABSTRACT: We calculate, using our recently proposed semiclassical framework, the quantum state of the Hawking pairs that are produced during the evaporation of a black hole $(\mathrm{BH})$. Our framework adheres to the standard rules of quantum mechanics and incorporates the quantum fluctuations of the collapsing shell spacetime in Hawking's original calculation, while accounting for back-reaction effects. We argue that the negative-energy Hawking modes need to be regularly integrated out; and so these are effectively subsumed by the $\mathrm{BH}$ and, as a result, the number of coherent negative-energy modes $N_{c o h}$ at any given time is parametrically smaller than the total number of the Hawking particles $N_{\text {total }}$ emitted during the lifetime of the $\mathrm{BH}$. We find that $N_{c o h}$ is determined by the width of the $\mathrm{BH}$ wavefunction and scales as the square root of the $\mathrm{BH}$ entropy. We also find that the coherent negative-energy modes are strongly entangled with their positive-energy partners. Previously, we have found that $N_{c o h}$ is also the number of coherent outgoing particles and that information can be continually transferred to the outgoing radiation at a rate set by $N_{c o h}$. Our current results show that, while the BH is semiclassical, information can be released without jeopardizing the nearly maximal inside-out entanglement and imply that the state of matter near the horizon is approximately the vacuum. The $\mathrm{BH}$ firewall proposal, on the other hand, is that the state of matter near the horizon deviates substantially from the vacuum, starting at the Page time. We find that, under the usual assumptions for justifying the formation of a firewall, one does indeed form at the Page time. However, the possible loophole lies in the implicit assumption that the number of strongly entangled pairs can be of the same order of $N_{\text {total }}$.

KEYwords: Black Holes, Models of Quantum Gravity

ARXIV EPRINT: 1312.0880 


\section{Contents}

1 Introduction $\quad 1$

1.1 The case against firewalls 5

2 Review of semiclassical black holes and the radiation density matrix $\quad 6$

2.1 Conventions 6

2.2 Time-dependent semiclassical radiation density matrix $\quad 7$

$\begin{array}{lll}3 & \text { Semiclassical state of the Hawking pairs } & 8\end{array}$

4 Model of semiclassical back-reaction $\quad 13$

5 The black hole Hilbert space $\quad 15$

$6 \quad$ Multi-pair density matrix $\quad 15$

$\begin{array}{lll}7 & \text { In-Out entanglement } & 19\end{array}$

$\begin{array}{lll}7.1 & \text { In-Out entanglement for } t<t_{\text {trans }} & 19\end{array}$

$\begin{array}{ll}\text { 7.2 Qualitative analysis of the in-out entanglement for } t>t_{\text {trans }} & 19\end{array}$

7.3 Summary of the evolution of entanglement entropy and released information 21

8 Discussion

\section{Introduction}

That a black hole $(\mathrm{BH})$ emits thermal radiation $[1,2]$ presents the following puzzle: How does an initially pure state of matter evolve into a mixed state of radiation without violating the principles of quantum mechanics? This is, in a nutshell, the BH information-loss paradox. (See [3] for Hawking's seminal discussion and [4-8] for reviews.)

Although this puzzle is regarded by many as an open question, most of the recent attention in this context has gone to a related issue that is known as the "firewall" paradox [9]. Also see [6-8, 10-12] for earlier versions of the same idea and [13-36] for what is just a sample of the ensuing discussion. From this new perspective, one assumes that the radiation does purify eventually and then asks what are the consequences to the standard picture of an observer falling harmlessly through the horizon. From an inspection of the literature, one finds that the answers range from nothing at all to the observer being set a blaze in a sea of high-energy quanta. Obviously, controversy abounds.

A simplified account of the firewall problem goes as follows: Let us parse the BH radiation into three subsystems; the "early" Hawking particles, the "late" Hawking particles and the interior "partners" of the late Hawking modes. Early and late in this context 
means before or after the so-called Page time [37,38], which is the midway point of entropy transfer. Following many, let us call the subsystems $A, B$ and $C$ respectively. Now, for the radiation to purify, $A$ and $B$ must be highly entangled. However, for an observer to fall through the horizon without trauma, $B$ must be close to maximally entangled with $C$. But this is a contradiction because of the monogamy-of-entanglement rule; no system can be simultaneously highly entangled with two different systems. And so, given that the purification of the radiation is true, $B$ and $C$ cannot be maximally entangled. Hence, the state of the near-horizon radiation differs substantially from the vacuum st ate and, therefore, the horizon must be a highly excited region that is filled with non-partnered quanta. As a consequence, the free-falling observer can expect to burn up on route or, put metaphorically, encounter a firewall.

See $[32,33]$ for a recent clarification of the proposal and for an additional discussion on monogamy of entanglement and purity in this context.

There has been a variety proposals for circumventing the firewall paradox, many of which have focused on the explicit assumptions in [9], which are based on the proposed tenets [39] of $\mathrm{BH}$ complementarity [40] and have already been countered by the original authors $[26,31]$. A relatively new development is the issue of state dependence in some of the proposed complementarity maps; see [32-35] in particular.

We would like to point out an implicit assumption that is being made by both the original paper and in many subsequent articles: Namely, that the number of paired Hawking modes is about the same as the total number of emitted Hawking particles up until (at least) the Page time. This assumption was made by Page in his original quantum-information treatment of a radiating system $[37,38]$ and is also made in all models for which the Page time is the moment when information becomes accessible. However, we will argue that, for an evaporating $\mathrm{BH}$, this assumption is not necessarily correct and its consequences should therefore be reconsidered.

The firewall argument relies on the standard description of BH evaporation as developed by Hawking [1-3]. The positive-energy Hawking modes and their negative-energy partners are created continuously throughout the evaporation process and accumulate near the horizon; just outside and just inside the horizon, respectively. The positive-energy modes escape to infinity, where they are observed as a thermal flux of radiation. The thermal nature of this radiation was established by Hawking from a direct calculation of the density matrix for the outgoing modes and does not require any knowledge about the ingoing partners. However, from the pair-production perspective, the thermal nature results from tracing over the negative-energy members of the maximally entangled pairs. The pairs are in a thermofield-double state; however, each of the pairs is produced in a process that is independent of the production of all the others, so that the pairs themselves are incohe rent.

Hawking's setup treats the BH as a strictly classical geometry. In [41], it was proposed that the $\mathrm{BH}$ information paradox originates from this assumption. (See [42-47] for overlapping ideas.) On the basis of [48, 49], it was also proposed in [41] that the leading semiclassical corrections resulting from quantum fluctuations of the background geometry should be taken into account by assigning a wavefunction to the $\mathrm{BH}$. The parameter that 
controls the strength of the semiclassical corrections was identified as the ratio of the Compton wavelength of the $\mathrm{BH} \quad \lambda_{B H}=\hbar / M_{B H}$ to its radial (Schwarzschild) size $R_{S}$. In [50], we have proposed a concrete scheme for evaluating the semiclassical corrections using the wavefunction of $[41,48]$. The parameter that controls the strength of the semiclassical corrections was denoted by $C_{B H}$ and calibrated more precisely, $C_{B H}=\frac{\lambda_{B H}}{2 \pi} / R_{S}=1 / S_{B H}$ $\left(S_{B H}\right.$ is the Bekenstein-Hawking entropy). This parameter can be viewed as a dimensionless $\hbar$ that is controlling quantum corrections.

We have, in two recent articles [51,52], gone on to apply this idea to the calculation of the Hawking radiation. There, Hawking's calculation was repeated by replacing the classical collapsing shell of matter (i.e., the incipient BH) with a semiclassical one that is endowed with a Gaussian wavefunction. The wavefunction introduces a new scale into the problem via its quantum width. The Bohr correspondence principle has been invoked to show that this width should be Planckian [41, 48].

We have recalculated the density matrix of the outgoing particles and obtained a picture that is similar to Hawking's in the limit $C_{B H}=0$. But, for a finite value of $C_{B H}$ (albeit, a very small one), our picture differs significantly from that found by Hawking. The Hawking density matrix for the $\mathrm{BH}$ radiation is strictly diagonal, whereas our matrix contains small off-diagonal elements of order $\sqrt{C_{B H}}$ in the same basis. The effect of these elements on the eigenvalues of the matrix is initially small; however, as the number $N$ of emitted particles grows, so does the changes to the eigenvalues.

Another important distinction is the degree of coherence of the radiating particles. In Hawking's case, the emitted particles are incoherent at any time. In our picture, the number of coherent particles at any given moment is finite and set by a scale that we refer to as the radiation coherence time $t_{c o h}$. Typically, $t_{c o h}=R_{S}^{2} / l_{p}$ (where $l_{p}$ is the Planck length) and the number of coherent Hawking particles $N_{c o h}$ is equal to the number of particles that are emitted over this time scale, $N_{c o h}=1 / \sqrt{C_{B H}}=\sqrt{S_{B H}}$. These estimates are accurate during most of the lifetime of the $\mathrm{BH}$ and become inaccurate only at the last stages of evaporation.

The appearance of a coherence time scale in our formalism is quite natural because of the following reasoning: The back-reaction of the emitted particles on the collapsing shell of matter leads to a time-dependent wavefunction. Let us then consider the time required for this wavefunction to change significantly. An inspection of its formal expression (see eq. (2.2)) indicates that this happens when the Schwarzschild radius shrinks by an amount $\Delta R_{S} \sim \sqrt{C_{B H}} R_{S} \sim l_{p}$. Then, since $\Delta R_{S}=\frac{\partial R_{S}}{\partial t} \Delta t \sim-\frac{l_{p}^{2}}{R_{S}^{2}} \Delta t$, it follows that $\Delta t \sim \frac{R_{S}^{2}}{l_{p}}=t_{c o h}$. Hence, the coherence time originates as the interval for which the overlap of the wavefunction at different times becomes small. The fact that $N_{c o h} \ll S_{B H}$ (equivalently, $t_{c o h} \ll \tau_{B H}$, where $\tau_{B H}$ is the $\mathrm{BH}$ lifetime) is a consequence of the width of the wavefunction being much smaller than the Schwarzschild radius. This hierarchy of scales can be attributed to the $\mathrm{BH}$ being semiclassical, $C_{B H} \ll 1$.

Both differences are directly related to treating the $\mathrm{BH}$ as a semiclassical quantum state rather than a classical geometry. The strength of the off-diagonal terms is the dimensionless width of the wavefunction $\sqrt{C_{B H}}$ and the coherence scale is related to the overlap of 
the wavefunction at different times. Thus, our explicit calculations strengthen the ideas expressed in [41] that treating the $\mathrm{BH}$ as a semiclassical state is an essential element in resolving many of the issues surrounding $\mathrm{BH}$ physics.

Our framework, as described in [52], incorporates the back-reaction of the emitted particles on the collapsing shell in addition to the shell's wavefunction. What we have found is that Hawking was correct in dismissing the effect of the back-reaction when the background is strictly classical. However, for our semi-classical framework, the back-reaction on the shell does become important.

In this paper, we extend our previous calculations to the negative-energy modes and try to learn about the implications of our semiclassical framework on the pair-production perspective of $\mathrm{BH}$ evaporation.

From the pair-production perspective, it is appropriate to integrate out the shell of matter [3]. This amounts to replacing the shell by an eternal BH geometry with specific boundary conditions for matter fields in this geometry. In Hawking's model, this replacement is approximately valid for all times. From the pair-production point of view, it looks as if the negative-energy modes are concurrently being subsumed into and annihilating with the $\mathrm{BH}$. Consequently, the mass of the $\mathrm{BH}$ is decaying with time at a rate dictated by the thermal emission of the $\mathrm{BH}$. When combined, these observations suggest a picture of the negative-energy modes being continually recycled at some approximately constant rate while the total number of positive-energy Hawking particles is steadily growing at a rate that is set by the thermal emission of the $\mathrm{BH}$.

In our framework, the replacement of the collapsing shell with the eternal BH geometry has limited validity. The identity of the negative-energy modes is not well defined and is sensitive to the decrease of the $\mathrm{BH}$ mass and radius due to the back-reaction. To resolve this issue of mode identities, we propose that, at regular intervals whose duration is one interval of coherence time, the shell spacetime has to be replaced by an eternal $\mathrm{BH}$ of smaller mass and smaller horizon radius.

The repetition of the process of integrating out the shell requires us to reassign the wavefunction to the $\mathrm{BH}$ and to redefine the pair basis accordingly. It follows that the negative-energy modes should be traced over at these regular intervals. The positiveenergy partners of the negative-energy modes that have been traced over then become part of the state of the external radiation.

It may appear that the process of regularly tracing over the negative-energy modes will lead to information loss. However, these anti-particles have actually been absorbed into the interior matter, and so the information about these modes is not lost but rather stored inside the BH. In subsection 7.3, we recall a qualitative discussion whose aim is to explain how the information could nevertheless be retrieved towards the end of the evaporation. For now, let us discuss an alternative point of view that will be elaborated on, quantitatively, in an upcoming article [53]: As the state of the $\mathrm{BH}$ is the purifier for the outgoing Hawking radiation, someone who is continuously monitoring the external radiation would know about the state of the $\mathrm{BH}$, including the subsumed anti-particles, as it evolves in time. Such an observer would then conclude that the radiation is monotonically purifying as the evaporation proceeds. 
The main objective of the current paper is to substantiate in a quantitative way the above description of $\mathrm{BH}$ evaporation in terms of the Hawking pairs. We calculate the quantum state of the in-out sector and show that the relevant pairs are in a state of nearly maximal entanglement, at least until the late stages of the evaporation process. But this is all that is needed because we have already shown that the early and late Hawking modes attain almost full entanglement but only at a similarly late time [51, 52].

The plan for the rest of the paper goes as follows: The subsequent section begins with a brief review of our previous results $[51,52]$. In section 3 , we reformulate our semiclassical density matrix for external radiation into a quantum state that describes the Hawking in-out modes. Next, in section 4, we elaborate on the above ideas about modeling the back-reaction and the need to trace out the negative-energy modes at regular intervals. The consequences of this model for the BH Hilbert space in the pair basis is the topic of section 5. We then construct a multi-pair density matrix in section 6 as a prelude to determining the entanglement entropy of the in-out sector. The latter calculation is carried out in section 7 . The paper concludes with a brief summary and discussion in section 8 .

\subsection{The case against firewalls}

Before proceeding, let us give a brief account of why our framework is able to evade the firewall problem without invoking changes to the standard rules of quantum mechanics.

As already mentioned, there is a hidden assumption in the current literature on firewalls that almost all of the produced in-modes are strongly entangled with their out-mode partners up until (at least) the Page time. After this, the external radiation is entropically dominant over the interior subsystem, which consists of the $\mathrm{BH}$ interior including the negative-energy modes; and so the status of these in-modes becomes a moot point. This is quite clear from the analysis of Page [37, 38] (also see [54]). In the total BH-radiation system, the larger of the two subsystems holds most of the system's entanglement in the form of internal correlations, rather than as a mutual entanglement with the smaller one. This is the significance of the Page time; the moment that the interior region and exterior particles exchange their previous roles as the dominant and submissive subsystem.

One can now see why a firewall is inevitable for the orthodox picture of evaporation. After the Page time, most of the entanglement is necessarily stored in correlations between early and late Hawking particles, these being the constituents of the dominant exterior subsystem. The now "unpartnered" in-modes will make the horizon a dangerous place.

But one can also recognize a possible loophole for evading the firewall problem. First consider that semiclassical deviations from maximal entanglement of the in-out pairs are controlled by the number of entangled in-out pairs. The deviations of the near-horizon state from the vacuum must therefore be controlled by this same number. Now suppose that the number of pairs is parametrically smaller than the total number of emitted Hawking particles. If so, then both the rate of information release and the degree of in-out entanglement will be controlled by the number of pairs rather than the size of the subsystems. What we have found is that the number of entangled pairs is equal to $N_{c o h}$, which is indeed parametrically smaller than the total number of emitted particles. If this possibility is realized, then there must be another component that purifies the outgoing radiation during 
most the lifetime of the $\mathrm{BH}$, otherwise unitarity will not be preserved. In our model, this component is the collapsed matter as represented by the shell and its wavefunction.

Because information is being released as the outgoing radiation purifies, there will come a time when the rate of information release is too large for the (nearly) maximal in-out entanglement to be maintained. At this point, assuming standard quantum mechanics, one could expect some large deviation of the near-horizon state from the vacuum and for the associated firewall to appear. What we find is that the new tipping point occurs parametrically close to the end of the evaporation, one interval of the coherence time before the $\mathrm{BH}$ totally evaporates. In [52], this is what we have called the transparency time $t_{\text {trans }}$. So that the Page time has, in effect, been moved to a time $t_{\text {trans }}$ that is late in the evaporation process. But, by this time, the $\mathrm{BH}$ can no longer be considered as semiclassical and there is no longer any good reason to expect its horizon to be a serene place (see below). How different is this late-time near-horizon state from the va cuum and what are its properties are interesting questions that we intend to answer in the future [55].

Let us explain why, at times $t>t_{\text {trans }}$, the $\mathrm{BH}$ can no longer be considered semiclassical even though it can still be macroscopically large with a near-horizon curvature that is small in Planck units. Our basic claim is that, for $t>t_{\text {trans }}$, an evaporating BH lacks a semiclassical description, irrespective of its size or the smallness of the curvature. The essential point is that the transparency time coincides with the time when $N_{c o h} C_{B H} \simeq$ 1 [52], meaning that $N_{c o h} \simeq S_{B H}$. Then, the number of negative-energy particles in the near-horizon region that are about to fall into the $\mathrm{BH}$ is about $S_{B H}$. It follows that their total energy is equal in magnitude to the energy of the remaining BH. Such a situation does not correspond anymore to the standard semiclassical picture of a large BH being weakly perturbed by a small number of negative-energy modes. Rather, back-reaction effects from the in-falling modes become large and significant, and so the notion of a nearly classical geometry for the $\mathrm{BH}$ is no longer tenable.

\section{Review of semiclassical black holes and the radiation density matrix}

Here, we review the results from our previous semiclassical model of the outgoing $\mathrm{BH}$ radiation. The framework was initially constructed in [51] and later improved upon in [52] by accounting for time-dependent and back-reaction effects.

\subsection{Conventions}

Our conventions are the same as in [52] and repeated here for completeness.

Our units are such that Planck's constant $\hbar$, Newton's constant $G$ or the combination $l_{p}=\sqrt{\hbar G}$ are explicit and all other fundamental constants are set to unity.

We assume a four-dimensional Schwarzschild BH (generalizations to higher dimensions are straightforward) of large but finite mass $M_{B H} \gg \sqrt{\hbar / G}$, with the metric $d s^{2}=-\left(1-\frac{R_{S}}{r}\right) d t^{2}+\left(1-\frac{R_{S}}{r}\right)^{-1} d r^{2}+r^{2} d \Omega_{2}^{2}$, where $R_{S}=2 G M_{B H}$ is the horizon radius. We use the dimensionless advanced-time coordinate $v=\frac{1}{R_{S}}\left(t+r^{*}\right)$, where $r^{*}=\int^{r} d r \sqrt{-g^{t t} g_{r r}}=r+R_{S} \ln \left(r-R_{S}\right)$. Our frequencies or $\omega$ 's are also dimensionless and measured in units of $1 / R_{S}$. 
The Hawking temperature $T_{H}$ and Bekenstein-Hawking entropy $S_{B H}$ of the $\mathrm{BH}$ are given by $T_{H}=\frac{\hbar}{4 \pi R_{S}}$ and $S_{B H}=\frac{\pi R_{S}^{2}}{\hbar G}$.

All classically evolving quantities (i.e., all functions of $\left.R_{S}=R_{S}(t)\right)$ should be regarded as time dependent.

\subsection{Time-dependent semiclassical radiation density matrix}

The meaning of the semiclassical density matrix, $\rho_{S C}=\rho_{H}+\Delta \rho_{S C}$, is the following:

$$
\rho_{S C}\left(\omega, \widetilde{\omega} ; C_{B H}\right)=\left\langle\Psi_{\text {shell }}\left(v_{\text {shell }}\right)|\rho(\omega, \widetilde{\omega})| \Psi_{\text {shell }}\left(v_{\text {shell }}\right)\right\rangle
$$

where $\Psi_{\text {shell }}\left(v_{\text {shell }}\right)$ is the wavefunction for the collapsing shell of matter. The diagonal Hawking density matrix $\rho_{H}$ picks up a correction $\Delta \rho_{S C} \sim \sqrt{C_{B H}}$ that introduces an off-diagonal modification.

We find $\Psi_{\text {shell }}\left(v_{\text {shell }}\right)$ by starting with the wavefunction for the $S$-mode of a Schwarzschild BH in Einstein gravity [41, 48, 50] and then assume that this describes the wavefunction of the shell in the limit of horizon formation, $R_{\text {shell }} \rightarrow R_{S}$ ( $R_{\text {shell }}$ is the shell's radius). This leads to

$$
\left.\Psi_{\text {shell }}\left(R_{\text {shell }}\right)\right|_{R_{\text {shell }} \rightarrow R_{S}}=\mathcal{N}^{-1 / 2} e^{-\frac{\left(R_{\text {shell }}-R_{S}\right)^{2}}{2 C_{B H} R_{S}^{2}}},
$$

where $\mathcal{N}$ is a normalization constant and $C_{B H}=S_{B H}^{-1}$ is the aforementioned classicality parameter. During most of the lifetime of the BH, when $C_{B H} \ll 1$, the spacetime can be treated as classical up to corrections going as a power series in $C_{B H}$. The correction $\Delta \rho_{S C}$ contains a factor $C_{B H}^{1 / 2}$ and so is suppressed relative to $\rho_{H}$. The classicality parameter $C_{B H}$ increases slowly and monotonically throughout the lifetime of the $\mathrm{BH}$ and trends to order unity when the size of the $\mathrm{BH}$ approaches the Planck scale.

Our prescription for calculating expectation values is

$$
\left\langle\widehat{O}\left(v_{\text {shell }}\right)\right\rangle=\frac{4 \pi}{\mathcal{N}} \int_{-\infty}^{\infty} d v_{\text {shell }} R_{\text {shell }}^{2}\left(v_{\text {shell }}\right) e^{-\frac{\left(v_{\text {shell }}-v_{0}\right)^{2}}{C_{B H}}} O\left(v_{\text {shell }}\right)
$$

where $v_{0}$ is the classical value of $v$ at horizon crossing, $\widehat{O}$ is a generic operator and we have used that $v_{0}-v \simeq R-R_{S}$ in the near-horizon limit.

Our later analysis in [52] entailed a time-dependent calculation that accounted for the different shell-crossing times of the Hawking modes and for the effect of the back-reaction on the shell. The number of emitted particles was found to be a good time coordinate, and eq. (2.1) gets corrected to

$$
\begin{aligned}
& \rho_{S C}\left(\omega, \widetilde{\omega} ; N_{T} ; N^{\prime}, N^{\prime \prime}\right)=\int_{-\infty}^{v_{0}} d v \int_{0}^{\infty} d \omega^{\prime} \int_{0}^{\infty} d \omega^{\prime \prime} \frac{1}{2 \pi} e^{i v\left(\omega^{\prime}-\omega^{\prime \prime}\right)} \\
& \quad \times e^{i \omega^{\prime}\left(v_{\text {shell }}\left(N_{T}\right)-v_{\text {shell }}\left(N^{\prime}\right)\right)} e^{-i \omega^{\prime \prime}\left(v_{\text {shell }}\left(N_{T}\right)-v_{\text {shell }}\left(N^{\prime \prime}\right)\right)} \\
& \times\left\langle\Psi_{\text {shell }}\left(v_{\text {shell }}\left(N_{T}\right)\right)\right| \beta_{\omega^{\prime} \omega, S C}^{*}\left(N_{T}\right) \beta_{\omega^{\prime \prime}} \widetilde{\omega}, S C \\
&
\end{aligned}
$$


where $N_{T}$ is the "time" since the $\mathrm{BH}$ formed, $N$ and $N^{\prime}$ are the respective shell-crossing times of a given pair of particles and the $\beta$ 's are Bogolubov coefficients. The subscript $S C$ on the $\beta$ 's indicates that these have been suitably reformulated in terms of the fluctuating parameter $v_{\text {shell }}$.

What we have found is that, after all integrations have been performed, the off-diagonal elements of the semiclassical density matrix pick up a time-dependent "suppression" factor $D\left(N_{T}, N^{\prime}, N^{\prime \prime}\right)$,

$$
\Delta \rho_{S C}\left(N_{T}, N^{\prime}, N^{\prime \prime}\right)=D\left(N_{T}, N^{\prime}, N^{\prime \prime}\right) \Delta \rho_{S C}\left(C_{B H}\left(N_{T}\right)\right) .
$$

where

$$
D\left(N_{T} ; N^{\prime}, N^{\prime \prime}\right) \equiv \frac{1}{2}\left[e^{-\frac{1}{4} \frac{\left[C_{B H}\left(N^{\prime}\right)\left(N_{T}-N^{\prime}\right)\right]^{2}}{C_{B H}\left(N_{T}\right)}}+e^{-\frac{1}{4} \frac{\left[C_{B H}\left(N^{\prime \prime}\right)\left(N_{T}-N^{\prime \prime}\right)\right]^{2}}{C_{B H}\left(N_{T}\right)}}\right]
$$

An important consequence of this factor is that our perturbative treatment - which formerly broke down at best by the Page time, $N_{T}=\frac{1}{2} S_{B H}(0)$ - can now be continued until much later in the process; essentially, until there are only $S_{B H}^{1 / 3}(0)$ particles remaining to be emitted (this being the transparency time).

The same suppression factor is obtained in the upcoming in-out treatment. It is still irrelevant to the Hawking part of the matrix, which already carries the implicit suppression $\delta\left(N^{\prime}-N^{\prime \prime}\right)$ because, in this case, the in- and out-modes emerge as perfectly entangled pairs.

The suppression is insignificant when $\Delta N \equiv N_{T}-N \leq N_{c o h}\left(N_{T} ; N\right)$, for which

$$
N_{c o h}\left(N_{T} ; N\right) \equiv \frac{\sqrt{C_{B H}\left(N_{T}\right)}}{C_{B H}(N)} .
$$

We call this the coherence time because, for $\Delta N>N_{c o h}\left(N_{T} ; N\right)$, the density matrix elements and, therefore, the particle correlations become highly suppressed. For most of the lifetime of the BH, $C_{B H}(N) \simeq C_{B H}\left(N_{T}\right)$, so that $N_{c o h}\left(N_{T} ; N\right) \simeq C_{B H}^{-1 / 2}\left(N_{T}\right) \simeq$ $\sqrt{S_{B H}\left(N_{T}\right)}$. At late times, however, this distinction can become important, as $C_{B H}\left(N_{T}\right)$ is monotonically growing as $\partial_{N_{T}} C_{B H}=C_{B H}^{2}$ and reaches unity for a Planck-sized BH.

\section{Semiclassical state of the Hawking pairs}

Before proceeding with the calculation of the pair semiclassical state, let us describe Hawking's pair-production picture and remark on some caveats.

Hawking's original choice of basis is that of the collapsing-shell model [1, 2]. For this choice, the negative-energy modes defy an obvious particle interpretation because, as far as an external observer is concerned, the separation between positive and negative energies becomes ambiguous inside of the shell's horizon. This ambiguity motivated Hawking to choose a different basis in his subsequent information-loss article [3]. This latter setup assumes an analytically continued Schwarzschild spacetime (i.e., an eternal BH geometry), for which such a separation can be made without ambiguity. In effect, to discuss the negative-energy modes, the shell is integrated out and replaced by an eternal BH geometry with a particular choice of boundary conditions for the matter fields. So that, in this model, the vicinity of the horizon is devoid of any matter. 
In principle, to calculate the semiclassical state for the pair-production model, we would need to know the wavefunction of the $\mathrm{BH}$ from an in-falling observer's perspective and then proceed along the lines of subsection 2.2. However, as explained below, we bypass this difficulty by exploiting a relationship between the in- and out-modes that allows us to use the external observer's wavefunction.

Hawking chose to work with the $w_{\omega}$ and $y_{\omega}$ basis of section 4 in [3], rather than the $q_{\omega}$ and $p_{\omega}$ basis of $[1,2]$. The $w$ 's are defined to have zero Cauchy data on $\mathcal{I}^{-}$and on the portion of the past horizon outside the future horizon. They represent particles that are always inside the future horizon. The $y$ 's are defined to have zero Cauchy data on $\mathcal{I}^{-}$ and on the portion of the past horizon inside the future horizon, as well as having positive energy with respect to the retarded time $u$ on the portion of the past horizon outside the future horizon. Hawking also showed that, as far as their action on the initial vacuum is concerned, the $y_{\omega}$ 's are equivalent to $p_{\omega}$ 's and the $w_{\omega}$ 's are equivalent to $p_{\omega}^{\dagger}$ 's.

The initial vacuum is defined at $\mathcal{I}^{-}$and the past horizon for the $w-y$ basis but only at $\mathcal{I}^{-}$for the $p-q$ basis. However, this distinction is irrelevant to the geometry of interest, the interior and exterior regions of the future horizon.

Hawking's choice of the $w-y$ basis corresponds to a partial tracing over some of the negative-energy modes (see below). We rather need to begin with modes that correspond to the complete "untraced" negative-energy modes. The complete horizon modes are denoted by Hawking as $f_{\omega}^{(3)}$ and $f_{\omega}^{(4)}$. They are defined as having zero Cauchy data on $\mathcal{I}^{-}$and, on the whole of the past horizon, they have time dependence of the form $e^{ \pm i \omega u}$, respectively. The operator forms of $f_{\omega}^{(3)}$ and $f_{\omega}^{(4)}$ are given in eq. (4.16) of [3] (re-expressed here in our notation),

$$
\begin{aligned}
& \widehat{f}_{\omega}^{(3)}=\frac{1}{\sqrt{1-c_{\omega}^{2}}}\left[\widehat{y}_{\omega}-c_{\omega} \widehat{w}_{-\omega}^{\dagger}\right], \\
& \widehat{f}_{\omega}^{(4)}=\frac{1}{\sqrt{1-c_{\omega}^{2}}}\left[\widehat{w}_{-\omega}-c_{\omega} \widehat{y}_{\omega}^{\dagger}\right],
\end{aligned}
$$

where $c_{\omega} \equiv e^{-2 \pi \omega}$.

This discussion highlights the fact that the pair-production picture has limited validity and is particularly sensitive to back-reaction induced deviations away from the eternal $\mathrm{BH}$ geometry. This sensitivity will be essential in the following.

Our eventual task is to calculate the entanglement between the out-modes - the incipient Hawking particles - and the in-modes - their negative-energy partners. For this calculation, we will first require the matrix elements for the final vacuum $\left|0_{+}\right\rangle$and then the expectation value of this matrix with respect to the initial vacuum $\left|0_{-}\right\rangle$. To this end, we will calculate the in-out analog of the out-out density matrix of our previous studies $[51,52]$. However, the resulting matrix $\rho_{\text {in-out }}$ should not be viewed as a density matrix but as the coefficients of the terms of an entangled state,

$$
\Psi_{\text {pair }}\left(\omega_{\text {out }},-\widetilde{\omega}_{\text {in }}\right)=\frac{1}{Z} \int d \omega d \widetilde{\omega} \rho_{\text {in-out }}\left(\omega_{\text {out }},-\widetilde{\omega}_{\text {in }}\right)\left|\omega_{\text {out }}\right\rangle\left|\widetilde{\omega}_{\text {in }}\right\rangle,
$$

where $Z$ is a normalization factor. 
The one-pair matrix that we have in mind is then the in-out analog of the following (with the expectation value implied on the left-hand side):

$$
\rho_{\text {out }- \text { out }}(\omega, \widetilde{\omega})=\left\langle 0_{-}\left|\left(\widehat{F}_{\omega}^{\dagger}+\widehat{F}_{\omega}\right)\right| 0_{+}\right\rangle\left\langle 0_{+}\left|\left(\widehat{F}_{\widetilde{\omega}}^{\dagger}+\widehat{F}_{\widetilde{\omega}}\right)\right| 0_{-}\right\rangle .
$$

Here, our notation is such that $\widehat{F}_{\omega}=F_{\omega}(u) \widehat{a}_{\omega}(F)$ includes both the wavefunction of the out-mode as a function of retarded time $u=u(v)$ and the annihilation operator. Analogous forms for other hatted modes are used below. We are currently considering a fixed value of advanced time $v$, but this coordinate is later integrated out.

It is a difficult task to calculate $\rho_{\text {in-out }}$ directly. As already stated, we would need to know the wavefunction of the horizon from an in-falling observer's perspective in the eternal $\mathrm{BH}$ geometry. Rather than doing this, we will express the in-modes in terms of a linear combination of the out-modes. We will then evaluate the corresponding $\rho_{\text {out-out }}$ and use the result to find $\rho_{\text {in-out }}$.

For the out-out case, one finds that the only relevant contribution of the four terms is $\widehat{F}_{\omega}^{\dagger} \widehat{F}_{\widetilde{\omega}}$, which leads to

$$
\rho_{\text {out }- \text { out }}(\omega, \widetilde{\omega})=\sum_{\omega^{\prime}, \omega^{\prime \prime}} f_{\omega^{\prime}}(v) \beta_{\omega^{\prime}, \omega}^{*} \beta_{\omega^{\prime \prime}, \widetilde{\omega}} f_{\omega^{\prime \prime}}^{*}(v)
$$

where the $\beta$ 's are the "negative-energy" Bogolubov coefficients and $f_{\omega^{\prime}}(v)$ is a basis function for the initial vacuum. The "positive-energy" Bogolubov coefficients or $\alpha$ 's enter through the other terms and could contribute in principle. However, as explained in subsection 2.4 of [51], these end up to be irrelevant for particle production for our semiclassical analysis just like in Hawking's treatment $[1,2]$.

Our out-modes are related to Hawking's modes in [3] as follows:

$$
\widehat{F}_{\omega}=t_{\omega} \widehat{y}_{\omega}+r_{\omega} \widehat{z}_{\omega}
$$

where $t_{\omega}$ and $r_{\omega}$ are the transmission and reflection coefficients, respectively. The mode $\widehat{z}_{\omega}$ is irrelevant for the pair-production process. Hence, for the purpose of calculating the in-out matrix, we can equate

$$
\widehat{y}_{\omega}=\frac{1}{t_{\omega}} \widehat{F}_{\omega} .
$$

To obtain the correct set of in- and out-modes for current purposes, we recall the following identity from [3]:

$$
\widehat{w}_{-\omega}^{\dagger}\left|0_{-}\right\rangle=c_{\omega}^{-1} \widehat{y}_{\omega}\left|0_{-}\right\rangle,
$$

where all frequencies are assigned according to the perspective of an external observer (i.e., $\omega>0$ in all cases). In other words, the creation of a negative-energy excitation is equivalent to the annihilation of a positive-energy one with the same magnitude of energy.

We propose that the correct definition for a complete horizon mode is as follows:

$$
\widehat{W}_{\omega}=\frac{1}{2}\left[\widehat{f}_{\omega}^{(3)}+\left(\widehat{f}_{\omega}^{(3)}\right)^{\dagger}+\widehat{f}_{\omega}^{(4)}+\left(\widehat{f}_{\omega}^{(4)}\right)^{\dagger}\right]
$$


where it is understood that only its negative-energy component contributes to the in-out density matrix as depicted in eq. (3.11) below. Then, using eqs. (3.1), (3.2) as well as eq. (3.8) to trade off $\widehat{w}_{\omega}$ 's for $\widehat{y}_{\omega}$ 's, we can express $\widehat{W}_{\omega}$ in terms of the out-mode $\widehat{y}_{\omega}$,

$$
\begin{aligned}
\widehat{W}_{\omega} & =\frac{1}{2} \frac{1}{\sqrt{1-c_{\omega}^{2}}}\left(1-c_{\omega}\right)\left(1+\frac{1}{c_{\omega}}\right)\left[\widehat{y}_{\omega}+\widehat{y}_{\omega}^{\dagger}\right] \\
& =\frac{1}{2} \frac{\sqrt{1-c_{\omega}^{2}}}{c_{\omega}}\left[\widehat{y}_{\omega}+\widehat{y}_{\omega}^{\dagger}\right] .
\end{aligned}
$$

The in-out matrix has now been expressed entirely in terms of out-modes,

$$
\begin{aligned}
\rho_{\text {in-out }}(\omega,-\widetilde{\omega}) & =\frac{1}{2}\left\langle 0_{-}\left|\left(\widehat{y}_{\omega}^{\dagger}+\widehat{y}_{\omega}\right)\right| 0_{+}\right\rangle\left\langle 0_{+}\left|\left(\widehat{W}_{\widetilde{\omega}}^{\dagger}+\widehat{W}_{\widetilde{\omega}}\right)\right| 0_{-}\right\rangle \\
& =\frac{\sqrt{1-c_{\widetilde{\omega}}^{2}}}{c_{\widetilde{\omega}}}\left\langle 0_{-}\left|\left(\widehat{y}_{\omega}^{\dagger}+\widehat{y}_{\omega}\right)\right| 0_{+}\right\rangle\left\langle 0_{+}\left|\left(\widehat{y}_{\widetilde{\omega}}+\widehat{y}_{\widetilde{\omega}}^{\dagger}\right)\right| 0_{-}\right\rangle \\
& =\frac{\sqrt{1-c_{\widetilde{\omega}}^{2}}}{c_{\widetilde{\omega}}}\left\langle 0_{-}\left|\left(\frac{1}{t_{\omega}^{*}} \widehat{F}_{\omega}^{\dagger}+\frac{1}{t_{\omega}} \widehat{F}_{\omega}\right)\right| 0_{+}\right\rangle\left\langle 0_{+}\left|\left(\frac{1}{t_{\widetilde{\omega}}} \widehat{F}_{\widetilde{\omega}}+\frac{1}{t_{\widetilde{\omega}}^{*}} \widehat{F}_{\widetilde{\omega}}^{\dagger}\right)\right| 0_{-}\right\rangle .
\end{aligned}
$$

Here, the operator $\widehat{F}_{\omega}^{\dagger}$ should be regarded as an excitation of a horizon mode and not that of an asymptotic Hawking particle.

Now, just as for the out-out case, the only contribution to the density matrix comes from the pair $\widehat{F}_{\omega}^{\dagger} \widehat{F}_{\widetilde{\omega}}$, as the rest have either rapidly oscillating phases or represent irrelevant non-propagating modes. It follows that

$$
\rho_{\text {in }- \text { out }}(\omega,-\widetilde{\omega})=\frac{\sqrt{1-c_{\widetilde{\omega}}^{2}}}{c_{\widetilde{\omega}}}\left\langle 0_{-}\left|\frac{1}{t_{\omega}^{*}} \widehat{F}_{\omega}^{\dagger}\right| 0_{+}\right\rangle\left\langle 0_{+}\left|\frac{1}{t_{\widetilde{\omega}}} \widehat{F}_{\widetilde{\omega}}\right| 0_{-}\right\rangle .
$$

Next, expanding the matrix in terms of the basis kets $\left|f_{\omega^{\prime}}\right\rangle$, we obtain

$$
\begin{aligned}
\rho_{\text {in-out }}(\omega,-\widetilde{\omega}) & =\frac{1}{t_{\omega}^{*} t_{\widetilde{\omega}}} \frac{\sqrt{1-c_{\widetilde{\omega}}^{2}}}{c_{\widetilde{\omega}}} \sum_{\omega^{\prime}, \omega^{\prime \prime}}\left\langle 0_{-} \mid f_{\omega^{\prime}}\right\rangle\left\langle f_{\omega^{\prime}}\left|\widehat{F}_{\omega}^{\dagger}\right| 0_{+}\right\rangle\left\langle 0_{+}\left|\widehat{F}_{\widetilde{\omega}}\right| f_{\omega^{\prime \prime}}\right\rangle\left\langle f_{\omega^{\prime \prime}} \mid 0_{-}\right\rangle \\
& =\frac{1}{t_{\omega}^{*} t_{\widetilde{\omega}}} \frac{\sqrt{1-c_{\widetilde{\omega}}^{2}}}{c_{\widetilde{\omega}}} \sum_{\omega^{\prime}, \omega^{\prime \prime}} f_{\omega^{\prime}}(v)\left\langle f_{\omega^{\prime}} \mid F_{\omega}\right\rangle\left\langle F_{\widetilde{\omega}} \mid f_{\omega^{\prime \prime}}\right\rangle f_{\omega^{\prime \prime}}^{*}(v),
\end{aligned}
$$

where $\left|F_{\omega}\right\rangle$ means a one-particle ket.

It is the amplitudes in the last line that describe the overlap between particle modes and basis vectors and, therefore, represent the Bogolubov coefficients. Because the right-hand side of eq. (3.13) involves only out-modes, these coefficients are the same as those obtained in the out-out case. Hence, $\left\langle f_{\omega^{\prime}} \mid F_{\omega}\right\rangle=\beta_{\omega^{\prime}, \omega}^{*},\left\langle F_{\widetilde{\omega}} \mid f_{\omega^{\prime \prime}}\right\rangle=\beta_{\omega^{\prime \prime}, \widetilde{\omega}}$ and, consequently,

$$
\rho_{\text {in-out }}(\omega,-\widetilde{\omega})=\frac{1}{t_{\omega}^{*} t_{\widetilde{\omega}}} \frac{\sqrt{1-c_{\widetilde{\omega}}^{2}}}{c_{\widetilde{\omega}}} \sum_{\omega^{\prime}, \omega^{\prime \prime}} f_{\omega^{\prime}}(v) \beta_{\omega^{\prime}, \omega}^{*} \beta_{\omega^{\prime \prime}, \widetilde{\omega}} f_{\omega^{\prime \prime}}^{*}(v) .
$$


We have then ended up with a matrix that is similar in form to the matrix for the out-out case. Again, there is the Hawking classical-background contribution, except that it is now describing maximally entangled pure state of pairs and will lead to a thermal reduced density matrix, as explained below. It differs from the usual form by a factor which will turn out to be very significant,

$$
\left[\rho_{\text {in }- \text { out }}(\omega,-\widetilde{\omega})\right]_{H}=\frac{\sqrt{1-c_{\omega}^{2}}}{c_{\omega}} \frac{1}{e^{\frac{\hbar \omega}{T_{H}}}-1} \delta(\omega-\widetilde{\omega})
$$

where $T_{H} / \hbar=(4 \pi)^{-1}$ is the dimensionless Hawking temperature.

One formal difference between eq. (3.15) and Hawking's out-out matrix is that the transmission amplitudes of the out-modes through the gravitational barrier no longer appear. This is sensible because these are horizon modes and not the asymptotically transmitted Hawking particles.

A more important distinction is, however, the extra factor of

$$
\frac{\sqrt{1-c_{\omega}^{2}}}{c_{\omega}}=\frac{\sqrt{1-e^{-4 \pi \omega}}}{e^{-2 \pi \omega}}=\sqrt{e^{4 \pi \omega}-1}=\sqrt{e^{\frac{\hbar \omega}{T_{H}}}-1}
$$

In this way, we actually end up with the "square root" of the Hawking thermal form,

$$
\left[\rho_{\text {in }- \text { out }}(\omega,-\widetilde{\omega})\right]_{H}=\rho_{H}^{1 / 2}(\omega,-\widetilde{\omega}) \equiv \frac{1}{\sqrt{e^{\frac{\hbar \omega}{T_{H}}}-1}} \delta(\omega-\widetilde{\omega}) .
$$

Despite appearances, the matrix in eq. (3.17) is not a density matrix but, rather, represents a pure state. The state is a superposition of pairs with weights $\frac{1}{\sqrt{e^{\frac{\hbar \omega}{T_{H}}}-1}}$ and, therefore, a thermofield-double state for the pairs of positive- and negative-energy modes,

$$
\left|\Psi_{\text {pair }, H}\left(\omega_{\text {out }},-\widetilde{\omega}_{\text {in }}\right)\right\rangle=\frac{1}{Z} \int d \omega \frac{1}{\sqrt{e^{\frac{\hbar \omega}{T_{H}}}-1}}\left|\omega_{\text {out }}\right\rangle\left|\omega_{\text {in }}\right\rangle .
$$

Here, $\left|\omega_{\text {out }}\right\rangle$ denotes a positive-energy out-mode with frequency $\omega$ and $\left|\omega_{\text {in }}\right\rangle$, a negativeenergy in-mode with frequency $\omega$. The normalization factor $Z$ will be specified later on.

The full Hawking density matrix is given by

$$
\rho_{\text {pair }, H}\left(\omega_{\text {out }},-\widetilde{\omega}_{\text {in }}, \omega_{\text {out }}^{\prime},-\widetilde{\omega}_{\text {in }}^{\prime}\right)=\left|\Psi_{\text {pair }, H}\left(\omega_{\text {out }},-\widetilde{\omega}_{\text {in }}\right)\right\rangle\left\langle\Psi_{\text {pair }, H}\left(\omega_{\text {out }}^{\prime},-\widetilde{\omega}_{\text {in }}^{\prime}\right)\right| .
$$

The reduced matrix for the out-modes is obtained by tracing over the in-modes. So that, as standard for a thermofield-double state, the reduced matrix goes as the square of eq. (3.17) and the correct thermal matrix is indeed obtained.

Meanwhile, the same lengthy calculation as in $[51,52]$ will lead us to the semiclassical correction to the Hawking state,

$$
\Delta \rho_{S C}\left(\omega,-\widetilde{\omega} ; N_{T} ; N^{\prime}, N^{\prime \prime}\right)=D\left(N_{T} ; N^{\prime}, N^{\prime \prime}\right) C_{B H}^{1 / 2}\left(N_{T}\right) \Delta \rho_{O D}\left(\omega,-\widetilde{\omega} ; N_{T}\right)
$$


with

$$
\begin{aligned}
& \Delta \rho_{O D}\left(\omega,-\widetilde{\omega} ; N_{T}\right)=\frac{1}{(2 \pi)^{3}} \frac{2}{(\omega \widetilde{\omega})^{1 / 2}}\left(\frac{C_{B H}\left(N_{T}\right)}{4}\right)^{+2 i(\omega-\widetilde{\omega})} \\
& \quad \times \frac{\sqrt{e^{4 \pi \omega}-1}+\sqrt{e^{4 \pi \widetilde{\omega}}-1}}{2} \Gamma(1+2 i \omega) \Gamma(1-2 i \widetilde{\omega}) e^{-\pi(\omega+\widetilde{\omega})} \Gamma\left(\frac{1}{2}-i(\omega-\widetilde{\omega})\right) \\
& \quad \times\left\{\Gamma(2 i(\omega-\widetilde{\omega}))\left[\frac{\Gamma\left(\frac{1}{2}+2 i \widetilde{\omega}\right)}{\Gamma\left(\frac{1}{2}+2 i \omega\right)}+\frac{\Gamma\left(\frac{1}{2}-2 i \omega\right)}{\Gamma\left(\frac{1}{2}-2 i \widetilde{\omega}\right)}\right]+\frac{i}{\omega-\widetilde{\omega}}\right\},
\end{aligned}
$$

where we have symmetrized over the frequencies and have subtracted off a diagonal piece with the understanding that this acts as a small correction to the Hawking part of the matrix. The total number of so-far produced pairs $N_{T}$ is now keeping track of the evolution time and $N^{\prime}, N^{\prime \prime}$ are the pair-production times.

Equation (3.20) should be interpreted as a correction to the thermofield-double state of eq. (3.18). The correction means that the positive- and negative-energy modes are not exactly maximally entangled, with $C_{B H}^{1 / 2}$ controlling the deviation from maximal entanglement. This will be discussed in detail in section 6 .

\section{Model of semiclassical back-reaction}

In the previous section, we have highlighted the fact that the particle-pair picture requires one to integrate out the collapsing shell and use the geometry of the eternal $\mathrm{BH}$ with appropriate boundary conditions for matter fields. We have also emphasized that, as a consequence, the pair-production picture has limited validity and is particularly sensitive to deviations away from the eternal $\mathrm{BH}$ spacetime. We would now like to discuss this issue of validity in a more quantitative way and, in particular, determine the duration for which the eternal BH geometry is a good approximation to the collapsing-shell model. We will argue that this duration is $t_{c o h}$.

The issue of validity of the pair-production picture was not discussed in a meaningful way by Hawking because, in his calculation, the coherence time scale did not appear. Each pair emission was considered to be completely independent of the previous pairs. Hence, Hawking's choice of the eternal BH geometry had an exponentially small effect.

In the Introduction, we have briefly outlined a simple model for the back-reaction of the emitted particles when considering the pair-production picture. The basic idea is that the negative-energy members of the pairs should be regularly traced out as the mass of the $\mathrm{BH}$ decreases with time at a rate dictated by the thermal emission. This simple model can be made more precise as follows.

Let us consider the perspective of an external, stationary observer. Then, during one coherence time $t_{c o h}$, the BH emits $N_{c o h} \sim S_{B H}^{1 / 2}$ Hawking particles and its energy decreases by $\Delta E_{\text {shell }} \sim N_{\text {coh }} T_{H} \sim M_{p}$, where $M_{p}=l_{p}^{-1}$ is the Planck mass. The radius of the $\mathrm{BH}$ will then shrink by $l_{p}$, and its wavefunction becomes much different than it was at earlier times. This restricts considerations to time intervals of duration $\Delta t<t_{c o h}$.

Over this time interval, the $\mathrm{BH}$ and the pair-produced particles, both positive- and negative-energy ones, are coherent. But, for time intervals in excess of $t_{c o h}$, the negative- 
energy particles should be traced over (see below), leaving an almost thermal (reduced) density matrix for a block of $N_{c o h}$ positive-energy particles with some small corrections of order $\sqrt{\hbar}$. After their negative-energy partners have disappeared, these should be regarded as emitted Hawking particles and, as such, will become part of the out-out radiation density matrix.

Our model for the back-reaction is quite simple and still needs to be improved by providing a more precise description of the interaction of the negative-energy modes with the BH. However, even at the current level of precision, it is already clear that the negativeenergy particles cannot keep their identity after an elapse of time $t_{c o h}$. This can be seen from the following argument.

Let us first recall of the form of the out-mode wavefunctions (with $\omega, u$, $v$ dimensionless),

$$
F_{\omega}(u(v)) \sim e^{i \omega u} \sim e^{i \omega \ln \left(v_{0}-v\right)}
$$

and similarly for the in-modes but with the argument in the logarithm reversed. Now consider that the positive-energy particles accumulate near the horizon but only on the outside, whereas the negative-energy particles accumulate on the inside. In Hawking's description, it is not stated how close these modes are to the horizon, so that the distance $\Delta v=v_{0}-v$ remains unspecified. But our case is different because of the uncertainty due to the quantum width of the wavefunction. The wavefunction has Planckian width, which implies that the width of either particle layer is about $\Delta v=l_{p} / R_{S} \quad$ (or $l_{p}$ in dimensional units). Our model also keeps track of the shrinking of the Schwarzschild radius or, equivalently, the decreasing value of $v_{0}$. After one coherence time, $v_{0}$ decreases by an amount of the same order, $\Delta v_{0} \sim l_{p} / R_{S}$.

For a time interval $\Delta t>t_{c o h}, v_{0}$ will have changed by an amount that is greater in magnitude than the width of the particle layers, $\Delta v_{0}>\Delta v$. At this point, the argument of the logarithm in eq. (4.1) and its in-mode analogue are likely to change sign. When such a sign flip does occur, it essentially exchanges the meaning of the mode from a positive to a negative-energy excitation (or vice versa). This is just like what would happen if a Rindler mode is switched from the right wedge to the left wedge of Rindler space. Time flows in the opposite direction in the left wedge, and so positive energies become negative. Meaning that, after the elapse of a coherence time, the splitting into positive and negative energies becomes ill defined and the identity of the near-horizon modes becomes uncertain.

To resolve this issue of mode identities, we propose that, after each coherence time, the eternal $\mathrm{BH}$ spacetime should be reset to a new eternal $\mathrm{BH}$ spacetime corresponding to the updated Schwarzschild radius $R_{S}\left(t+t_{c o h}\right) \simeq R_{S}(t)-l_{p}$. The pair basis has to be redefined accordingly and the whole process repeats itself after the elapse of the next interval of coherent time.

In general, this limitation on the use of the eternal BH geometry should be imposed when the back-reaction is taken into account, irrespective of whether the geometry is treated as classical or semiclassical. The only situation in which the negative-energy particles can preserve their identity is for a truly eternal BH geometry. However, if the 
coherence time vanishes (as it does for Hawking's model), it is not important that these modes preserve their identity for the purpose of calculating the in-out density matrix.

\section{The black hole Hilbert space}

Let us now discuss how our previous model for the back-reaction is relevant to the structure of the $\mathrm{BH}$ Hilbert space $\mathcal{H}_{B H}$ in the pair basis.

The Hilbert space of an evaporating $\mathrm{BH}$ will approximately factorize into two Hilbert spaces, $\quad \mathcal{H}_{B H} \sim \mathcal{H}_{\text {int }} \otimes \mathcal{H}_{\text {rad }}$. Here, $\mathcal{H}_{\text {int }}$ describes the state of the collapsed matter plus the anti-particles and $\mathcal{H}_{\text {rad }}$ describes the outgoing Hawking radiation. The radiation in-out entangled sector is then the boundary or overlap between these two sub-Hilbert spaces, $\mathcal{H}_{\text {in-out }}=\mathcal{H}_{\text {int }} \bigcap \mathcal{H}_{\text {rad }}$. We know from the analysis of Page that this overlap is small compared to $S_{B H}$ because most of the entanglement is stored as internal correlations within the subsystems and not as correlations between the subsystems. But how small?

Our formalism suggests a definitive answer to this last question. As we now know, it is necessary to trace over the negative-energy modes after a time scale of $t_{c o h}$ or $N_{c o h} \simeq S_{B H}^{1 / 2}$ in units of either number of emitted particles or number of produced pairs. Our explanation is that this effect is a consequence of the wavefunction decohering over the same extent of time. The coherence scale is then the span of time over which it still makes sense to talk about entangled partners; meaning that $N_{c o h} \simeq S_{B H}^{1 / 2}$ is the typical lifetime of a partnership. This leads to a revision of the orthodox picture: Partnerships are being regularly dissolved and recycled as the Hawking process of mode creation goes on [1, 2], with the total number of entangled partners scaling as $N_{c o h}$. In short, we are arguing that $\operatorname{dim}\left[\mathcal{H}_{\text {in-out }}\right]=N_{\text {coh }}$.

This recycling process, over the time scale $N_{c o h} \ll S_{B H}$, provides the means for maintaining the in-out entanglement while information is flowing out of the $\mathrm{BH}$. That the entanglement is maintained will be clarified in the upcoming analysis, but then what is the mechanism for information transfer? It is the wavefunction $\Psi_{B H}$ that plays the role of conduit. As the in-modes are traced out and effectively subsumed into the BH interior, the Schwarzschild radius $R_{S}=R_{S}\left(N_{T}\right)$ decreases and, in turn, induces an evolving value for the coherence scale $N_{c o h}=N_{c o h}\left(N_{T} ; N\right)$.

An external stationary observer has direct access to $\mathcal{H}_{\text {rad }}$, whereas a free-falling observer is able to probe $\mathcal{H}_{\text {int }}$ but at the cost of relinquishing knowledge about the exterior system $\mathcal{H}_{\text {rad }}$. If such observers wish to compare measurements, their only common ground is that of the boundary region $\mathcal{H}_{\text {in-out }}$. We would like to suggest that this could be a starting point for a definition of $\mathrm{BH}$ complementarity $[39,40]$ that can survive the firewall paradox.

\section{Multi-pair density matrix}

To monitor the entanglement of the produced pairs — which is the subject of section 7 - it is first necessary to construct a multi-pair density matrix for the in-out sector. In light of the previous two sections, it is clear that the multi-pair matrix should involve 
$N_{c o h}$ pairs of particles. Then, following our earlier investigations [51, 52], the multipair matrix is a $2 N_{c o h} \times 2 N_{c o h}$ matrix such that each entry is a block with the same dimensionality in frequency space as the one-pair matrix, $\rho_{S C}\left(\omega,-\widetilde{\omega} ; N_{T} ; N^{\prime}, N^{\prime \prime}\right)=$ $\rho_{H}^{1 / 2}(\omega,-\widetilde{\omega})+\Delta \rho_{S C}\left(\omega,-\widetilde{\omega} ; N_{T} ; N^{\prime}, N^{\prime \prime}\right)$. Recall that $\rho_{H}^{1 / 2}(\omega,-\widetilde{\omega})$ is defined in eq. (3.17) and the correction in eqs. (3.20)-(3.21). The suppression factor $D\left(N_{T} ; N^{\prime}, N^{\prime \prime}\right)$ that appears in $\Delta \rho_{S C}$ is defined in eq. (2.6) and can be re-expressed in a convenient way,

$$
D\left(N_{T} ; N^{\prime}, N^{\prime \prime}\right)=\frac{1}{2}\left[e^{-\frac{1}{4} \frac{\left(N_{T}-N^{\prime}\right)^{2}}{N_{c o h}^{2}\left(N_{T} ; N^{\prime}\right)}}+e^{-\frac{1}{4} \frac{\left(N_{T}-N^{\prime \prime}\right)^{2}}{N_{c o h}^{2}\left(N_{T} ; N^{\prime \prime}\right)}}\right] .
$$

As discussed in $[51,52]$, one can expect each entry to pick up a phase factor $e^{i \theta_{N^{\prime}, N^{\prime \prime}}}$ $\left(\theta_{N^{\prime}, N^{\prime \prime}}=-\theta_{N^{\prime}, N^{\prime \prime}}\right)$. But these phases are not relevant to our treatment and will be ignored.

We can express the multi-particle (MP) state $\Psi_{S C}^{M P}\left(N_{T} ; N^{\prime}, N^{\prime \prime}\right)$ in Dirac notation (with frequency labels now suppressed),

$$
\begin{aligned}
\left|\Psi_{S C}^{M P}\left(N_{T} ; N^{\prime}, N^{\prime \prime}\right)\right\rangle & =\frac{1}{n_{*}} \rho_{H}^{1 / 2} \delta_{N^{\prime}, N^{\prime \prime}}\left|N^{\prime}\right\rangle\left|N^{\prime \prime}\right\rangle \\
& +\frac{C_{B H}^{1 / 2}\left(N_{T}\right)}{n_{*}} \Delta \rho_{O D} D\left(N_{T} ; N^{\prime}, N^{\prime \prime}\right)\left[1-\delta_{N^{\prime}, N^{\prime \prime}}\right]\left|N^{\prime}\right\rangle\left|N^{\prime \prime}\right\rangle,
\end{aligned}
$$

where $N_{T}-N_{c o h} \lesssim N^{\prime}, N^{\prime \prime} \leq N_{T}$. The normalization $n_{*}$ will be determined later on by the requirement that the reduced out-out density matrix be correctly normalized.

The density matrix $\rho_{S C}^{M P}$ corresponding to $\left|\Psi_{S C}^{M P}\right\rangle$ is given by the standard expression,

$$
\rho_{S C}^{M P}\left(N_{T} ; N^{\prime}, N^{\prime \prime}, N^{\prime \prime \prime}, N^{\prime \prime \prime \prime}\right)=\left|\Psi_{S C}^{M P}\left(N_{T} ; N^{\prime}, N^{\prime \prime}\right)\right\rangle\left\langle\Psi_{S C}^{M P}\left(N_{T} ; N^{\prime \prime \prime}, N^{\prime \prime \prime \prime}\right)\right| .
$$

To obtain a reduced density matrix for the out-modes, we need to re-express the density matrix on the product space $\left|N_{o}\right\rangle \otimes\left|N_{i}\right\rangle$. Then, $\rho_{S C, o \otimes i}=\rho_{S C, o \otimes i}\left(N_{T} ; N_{o}^{\prime}, N_{i}^{\prime}, N_{o}^{\prime \prime}, N_{i}^{\prime \prime}\right)$, where the subscripts $i$ and $o$ respectively label in- and out-modes. This matrix takes the form

$$
\begin{aligned}
& \rho_{S C, o \otimes i}\left(N_{T} ; N_{o}^{\prime}, N_{i}^{\prime}, N_{o}^{\prime \prime}, N_{i}^{\prime \prime}\right)=\frac{1}{n_{*}^{2}} \rho_{H}^{1 / 2} \otimes \rho_{H}^{1 / 2}\left|N_{o}^{\prime}\right\rangle\left|N_{i}^{\prime}\right\rangle\left\langle N_{o}^{\prime \prime}\right|\left\langle N_{i}^{\prime \prime}\right|\left[\delta_{N_{o}^{\prime}, N_{i}^{\prime}} \delta_{N_{o}^{\prime \prime}, N_{i}^{\prime \prime}}+\delta_{N_{o}^{\prime}, N_{i}^{\prime \prime}} \delta_{N_{o}^{\prime}, N_{i}^{\prime \prime}}\right] \\
& +\frac{C_{B H}\left(N_{T}\right)}{n_{*}^{2}} \Delta \rho_{O D} \otimes\left(\Delta \rho_{O D}\right)^{\dagger} \times\{ \\
& \quad D\left(N_{T} ; N_{o}^{\prime}, N_{i}^{\prime}\right) D\left(N_{T} ; N_{o}^{\prime \prime}, N_{i}^{\prime \prime}\right)\left|N_{o}^{\prime}\right\rangle\left|N_{i}^{\prime}\right\rangle\left\langle N_{o}^{\prime \prime}\right|\left\langle\left. N_{i}^{\prime \prime}\right|_{\left(N_{o}^{\prime} \neq N_{i}^{\prime}, N_{o}^{\prime \prime} \neq N_{i}^{\prime \prime}\right)}\right. \\
& +D\left(N_{T} ; N_{o}^{\prime}, N_{i}^{\prime \prime}\right) D\left(N_{T} ; N_{o}^{\prime \prime}, N_{i}^{\prime}\right)\left|N_{o}^{\prime}\right\rangle\left|N_{i}^{\prime}\right\rangle\left\langle N_{o}^{\prime \prime}\right|\left\langle\left. N_{i}^{\prime \prime}\right|_{\left(N_{o}^{\prime} \neq N_{i}^{\prime \prime}, N_{o}^{\prime \prime} \neq N_{i}^{\prime}\right)}\right\} \\
& +\frac{C_{B H}^{1 / 2}\left(N_{T}\right)}{n_{*}^{2}} \frac{1}{2}\left[\Delta \rho_{O D} \otimes \rho_{H}^{1 / 2}+\rho_{H}^{1 / 2} \otimes\left(\Delta \rho_{O D}\right)^{\dagger}\right] \times\{ \\
& +D\left(N_{T} ; N_{o}^{\prime}, N_{i}^{\prime}\right) \delta_{N_{o}^{\prime \prime}, N_{i}^{\prime \prime}}\left|N_{o}^{\prime}\right\rangle\left|N_{i}^{\prime}\right\rangle\left\langle N_{o}^{\prime \prime}\right|\left\langle\left. N_{i}^{\prime \prime}\right|_{N_{o}^{\prime} \neq N_{i}^{\prime}}\right. \\
& +D\left(N_{T} ; N_{o}^{\prime}, N_{i}^{\prime \prime}\right) \delta_{N_{o}^{\prime \prime}, N_{i}^{\prime}}\left|N_{o}^{\prime}\right\rangle\left|N_{i}^{\prime}\right\rangle\left\langle N_{o}^{\prime \prime}\right|\left\langle\left. N_{i}^{\prime \prime}\right|_{N_{o}^{\prime} \neq N_{i}^{\prime \prime}}\right. \\
& +D\left(N_{T} ; N_{o}^{\prime \prime}, N_{i}^{\prime}\right) \delta_{N_{o}^{\prime}, N_{i}^{\prime \prime}}\left|N_{o}^{\prime}\right\rangle\left|N_{i}^{\prime}\right\rangle\left\langle N_{o}^{\prime \prime}\right|\left\langle\left. N_{i}^{\prime \prime}\right|_{N_{o}^{\prime \prime} \neq N_{i}^{\prime}}\right. \\
& +D\left(N_{T} ; N_{o}^{\prime \prime}, N_{i}^{\prime \prime}\right) \delta_{N_{o}^{\prime}, N_{i}^{\prime}}\left|N_{o}^{\prime}\right\rangle\left|N_{i}^{\prime}\right\rangle\left\langle N_{o}^{\prime \prime}\right|\left\langle\left. N_{i}^{\prime \prime}\right|_{N_{o}^{\prime \prime} \neq N_{i}^{\prime \prime}}\right\}
\end{aligned}
$$

where $\rho_{H}^{1 / 2} \otimes \rho_{H}^{1 / 2} \quad$ denotes, respectively, $\rho_{H}^{1 / 2}\left(\omega_{o^{\prime}},-\widetilde{\omega}_{i^{\prime}}\right) \otimes \rho_{H}^{1 / 2}\left(\omega_{o^{\prime \prime}},-\widetilde{\omega}_{i^{\prime \prime}}\right)$, $\rho_{H}^{1 / 2}\left(\omega_{o^{\prime}},-\widetilde{\omega}_{i^{\prime \prime}}\right) \otimes \rho_{H}^{1 / 2}\left(\omega_{o^{\prime \prime}},-\widetilde{\omega}_{i^{\prime}}\right)$ and $\Delta \rho_{O D} \otimes\left(\Delta \rho_{O D}\right)^{\dagger}$ denotes, respectively, 
$\frac{1}{2}\left[\Delta \rho_{O D}\left(\omega_{o^{\prime}},-\widetilde{\omega}_{i^{\prime}}\right) \otimes \Delta \rho_{O D}^{\dagger}\left(\omega_{o^{\prime \prime}},-\widetilde{\omega}_{i^{\prime \prime}}\right)+\Delta \rho_{O D}\left(\omega_{o^{\prime \prime}},-\widetilde{\omega}_{i^{\prime \prime}}\right) \otimes \Delta \rho_{O D}^{\dagger}\left(\omega_{O^{\prime}},-\widetilde{\omega}_{i^{\prime}}\right)\right]$,
$\frac{1}{2}\left[\Delta \rho_{O D}\left(\omega_{o^{\prime}}, \widetilde{\omega}_{i^{\prime}}\right) \otimes \Delta \rho_{O D}^{\dagger}\left(\omega_{o^{\prime \prime}},-\widetilde{\omega}_{i^{\prime}}\right)+\Delta \rho_{O D}\left(\omega_{o^{\prime \prime}},-\widetilde{\omega}_{i^{\prime}}\right) \otimes \Delta \rho_{O D}^{\dagger}\left(\omega_{o^{\prime}},-\widetilde{\omega}_{i^{\prime \prime}}\right)\right]$.

$2\left[\Delta \rho_{O D}\left(\omega_{O^{\prime}},-\widetilde{\omega}_{i^{\prime \prime}}\right) \otimes \Delta \rho_{O D}^{\dagger}\left(\omega_{O^{\prime \prime}},-\widetilde{\omega}_{i^{\prime}}\right)+\Delta \rho_{O D}\left(\omega_{O^{\prime \prime}},-\widetilde{\omega}_{i^{\prime}}\right) \otimes \Delta \rho_{O D}^{\dagger}\left(\omega_{O^{\prime}},-\widetilde{\omega}_{i^{\prime \prime}}\right)\right] \cdot$
expression $\frac{1}{2}\left[\Delta \rho_{O D} \otimes \rho_{H}^{1 / 2}+\rho_{H}^{1 / 2} \otimes\left(\Delta \rho_{O D}\right)^{\dagger}\right] \quad$ denotes, respectively, the following: $\quad \frac{1}{2}\left[\Delta \rho_{O D}\left(\omega_{o^{\prime}},-\widetilde{\omega}_{i^{\prime}}\right) \otimes \rho_{H}^{1 / 2}\left(\omega_{0^{\prime \prime}},-\widetilde{\omega}_{i^{\prime \prime}}\right)+\rho_{H}^{1 / 2}\left(\omega_{o^{\prime \prime}},-\widetilde{\omega}_{i^{\prime \prime}}\right) \otimes \Delta \rho_{O D}^{\dagger}\left(\omega_{o^{\prime}},-\widetilde{\omega}_{i^{\prime}}\right)\right]$,

$\frac{1}{2}\left[\Delta \rho_{O D}\left(\omega_{o^{\prime}},-\widetilde{\omega}_{i^{\prime \prime}}\right) \otimes \rho_{H}^{1 / 2}\left(\omega_{o^{\prime \prime}},-\widetilde{\omega}_{i^{\prime}}\right)+\rho_{H}^{1 / 2}\left(\omega_{o^{\prime \prime}},-\widetilde{\omega}_{i^{\prime}}\right) \otimes \Delta \rho_{O D}^{\dagger}\left(\omega_{o^{\prime}},-\widetilde{\omega}_{i^{\prime \prime}}\right)\right]$,

$\frac{1}{2}\left[\Delta \rho_{O D}\left(\omega_{o^{\prime \prime}},-\widetilde{\omega}_{i^{\prime}}\right) \otimes \rho_{H}^{1 / 2}\left(\omega_{O^{\prime}},-\widetilde{\omega}_{i^{\prime \prime}}\right)+\rho_{H}^{1 / 2}\left(\omega_{O^{\prime}},-\widetilde{\omega}_{i^{\prime \prime}}\right) \otimes \Delta \rho_{O D}^{\dagger}\left(\omega_{o^{\prime \prime}},-\widetilde{\omega}_{i^{\prime}}\right)\right]$,

$\frac{1}{2}\left[\Delta \rho_{O D}\left(\omega_{o^{\prime \prime}},-\widetilde{\omega}_{i^{\prime \prime}}\right) \otimes \rho_{H}^{1 / 2}\left(\omega_{o^{\prime}},-\widetilde{\omega}_{i^{\prime}}\right)+\rho_{H}^{1 / 2}\left(\omega_{o^{\prime}},-\widetilde{\omega}_{i^{\prime}}\right) \otimes \Delta \rho_{O D}^{\dagger}\left(\omega_{o^{\prime \prime}},-\widetilde{\omega}_{i^{\prime \prime}}\right)\right]$.

The out-particle reduced density matrix is obtained by tracing over the frequencies and particle numbers of the in-mode Hilbert space, $\rho_{S C, \text { out }}=\operatorname{Tr}_{\text {in }} \rho_{S C, o \otimes i}$. This is a straightforward calculation for the Hawking part of the matrix. For the correction, it entails computing the integral

$$
\mathcal{I}_{b}=\int_{N_{T}-N_{c o h}\left(N_{T}\right)}^{N_{T}} d N_{i} e^{-\frac{1}{4} b \frac{\left(N_{T}-N_{i}\right)^{2}}{N_{c o h}^{2}\left(N_{T} ; N_{i}\right)}}
$$

where $N_{c o h}\left(N_{T} ; N_{i}\right) \gg 1$ allows us to treat the discrete sum as continuous and $b$ is either 0,1 or 2 . For the terms of order $C_{B H}^{1 / 2}$, then $b$ is 0 or 1 and, for the terms of order $C_{B H}$, then $b$ depends on which of the four different products of exponents is being considered in the product of suppression factors,

$$
\frac{1}{4}\left[e^{-\frac{1}{4} \frac{\left(N_{T}-N_{o}^{\prime}\right)^{2}}{N_{c o h}^{2}\left(N_{T} ; N_{o}^{\prime}\right)}}+e^{-\frac{1}{4} \frac{\left(N_{T}-N_{i}\right)^{2}}{N_{c o h}^{2}\left(N_{T} ; N_{i}\right)}}\right] \times\left[e^{-\frac{1}{4} \frac{\left(N_{T}-N_{o}^{\prime \prime}\right)^{2}}{N_{c o h}^{2}\left(N_{T} ; N_{o}^{\prime \prime}\right)}}+e^{-\frac{1}{4} \frac{\left(N_{T}-N_{i}\right)^{2}}{N_{c o h}^{2}\left(N_{T} ; N_{i}\right)}}\right] .
$$

We will consider, for the most part, "typical" times $t$ such that $t_{c o h}<t<\tau_{B H}-t_{c o h}$. Then the dependence on the second argument in $N_{\text {coh }}\left(N_{T} ; N_{i}\right)$ is weak, $N_{c o h}\left(N_{T} ; N_{i}\right) \simeq$ $N_{c o h}\left(N_{T}\right) \equiv N_{c o h}\left(N_{T} ; N_{T}\right)$ for the relevant values of $N_{i}$. This is made clear in the next paragraph.

It is obvious that $\mathcal{I}_{b=0}=N_{c o h}\left(N_{T}\right)$. For $b=1,2$, we note that $C_{B H}\left(N_{T}\right)$ is approximately constant over one interval of coherence time. This is because $C_{B H}\left(N_{T}\right) \simeq$ $\left[S_{B H}(0)-N_{T}\right]^{-1}[51]$, from which it follows that the change of $C_{B H}$ over a coherence time is small, $\Delta C_{B H} \simeq \frac{\partial C_{B H}}{\partial N_{T}} N_{c o h} \simeq C_{B H}^{2} N_{c o h} \ll 1$. Consequently, the integrand in $\mathcal{I}_{b=1,2}$ is approximately unity over the range of integration. It can be concluded that $\mathcal{I}_{b} \simeq N_{c o h}\left(N_{T}\right)$ for $b=0,1,2$.

It is now straightforward to evaluate the reduced density matrix,

$$
\begin{aligned}
\rho_{S C, \text { out }}\left(N_{T} ; N_{o}^{\prime}, N_{o}^{\prime \prime}\right) & =\frac{2 N_{c o h}\left(N_{T}\right)}{n_{*}^{2}} \operatorname{Tr}_{\mathrm{in}}\left[\rho_{H}\right]\left|N_{o}^{\prime}\right\rangle\left\langle N_{o}^{\prime \prime}\right| \delta_{N_{o}^{\prime}, N_{o}^{\prime \prime}} \\
& +C_{B H}\left(N_{T}\right) \frac{N_{c o h}\left(N_{T}\right)}{2 n_{*}^{2}} \operatorname{Tr}_{\mathrm{in}}\left[\left|\Delta \rho_{O D}\right|^{2}\right] \widetilde{D}\left(N_{T} ; N_{o}^{\prime}, N_{o}^{\prime \prime}\right)\left|N_{o}^{\prime}\right\rangle\left\langle N_{o}^{\prime \prime}\right| \\
& +C_{B H}^{1 / 2}\left(N_{T}\right) \frac{N_{c o h}\left(N_{T}\right)}{n_{*}^{2}} \operatorname{Tr}_{\mathrm{in}}\left[\overline{\Delta \rho}_{O D}\right] \bar{D}\left(N_{T} ; N_{o}^{\prime}, N_{o}^{\prime \prime}\right)\left|N_{o}^{\prime}\right\rangle\left\langle\left. N_{o}^{\prime \prime}\right|_{N_{o}^{\prime} \neq N_{o}^{\prime \prime}},\right.
\end{aligned}
$$


where

$$
\begin{aligned}
& \widetilde{D}\left(N_{T} ; N^{\prime}, N^{\prime \prime}\right) \equiv\left[1+e^{-\frac{1}{4} \frac{\left[C_{B H}\left(N^{\prime}\right)\left(N_{T}-N^{\prime}\right)\right]^{2}}{C_{B H}\left(N_{T}\right)}}\right] \times\left[1+e^{-\frac{1}{4} \frac{\left[C_{B H}\left(N^{\prime \prime}\right)\left(N_{T}-N^{\prime \prime}\right)\right]^{2}}{C_{B H}\left(N_{T}\right)}}\right], \\
& \bar{D}\left(N_{T} ; N^{\prime}, N^{\prime \prime}\right) \equiv e^{-\frac{1}{4} \frac{\left[C_{B H}\left(N^{\prime}\right)\left(N_{T}-N^{\prime}\right)\right]^{2}}{C_{B H}\left(N_{T}\right)}}+2+e^{-\frac{1}{4} \frac{\left[C_{B H}\left(N^{\prime \prime}\right)\left(N_{T}-N^{\prime \prime}\right)\right]^{2}}{C_{B H}\left(N_{T}\right)}}, \\
& \overline{\Delta \rho}_{O D}=\frac{1}{2}\left[\Delta \rho_{O D} \otimes \rho_{H}^{1 / 2}+\rho_{H}^{1 / 2} \otimes\left(\Delta \rho_{O D}\right)^{\dagger}\right]
\end{aligned}
$$

and $\operatorname{Tr}_{\text {in }}\left[\rho\left(\omega_{o^{\prime}},-\widetilde{\omega}_{i^{\prime}}\right) \rho^{\dagger}\left(\omega_{o^{\prime \prime}},-\widetilde{\omega}_{i^{\prime \prime}}\right)\right]=\int_{0}^{\infty} d x \rho\left(\omega_{o^{\prime}},-x\right) \rho^{\dagger}\left(\omega_{o^{\prime \prime}},-x\right)$. Here, $\rho$ means any single-pair matrix and $\operatorname{Tr}_{\text {in }}\left[\rho_{H}^{1 / 2} \rho_{H}^{1 / 2}\right]=\operatorname{Tr}_{\text {in }}\left[\rho_{H}\right]$ has been used.

To fix the normalization constant $n_{*}$ in eq. (6.7), we need to calculate the trace of $\rho_{S C \text {, out }}$. We will assume the convention that the full trace over the single-pair Hawking matrix gives unity, $\operatorname{Tr}_{\text {out }} \operatorname{Tr}_{\text {in }}\left[\rho_{H}\right]=1$, thus absorbing the implicit correction of order $C_{B H}^{1 / 2}$ for the diagonal $\left(N^{\prime}=N^{\prime \prime}\right)$ terms into the normalization.

The trace of the first term in $\rho_{S C}$, out is given by

$$
\frac{2 N_{c o h}\left(N_{T}\right)}{n_{*}^{2}} \operatorname{Tr}_{\text {out }}\left[\operatorname{Tr}_{\text {in }}\left[\rho_{H}\right]\left|N_{o}^{\prime}\right\rangle\left\langle N_{o}^{\prime \prime}\right| \delta_{N_{o}^{\prime}, N_{o}^{\prime \prime}}\right]=\frac{2 N_{c o h}^{2}\left(N_{T}\right)}{n_{*}^{2}} .
$$

For the calculation of the trace of the correction, the relevant integral is

$$
\mathcal{J}=\int_{N_{T}-N_{c o h}\left(N_{T}\right)}^{N_{T}} d N \widetilde{D}\left(N_{T} ; N ; N\right) .
$$

This integral has four contributions, each of which is of the same form as $\mathcal{I}_{b}$ in eq. (6.5). Then, by the same reasoning, $\mathcal{J} \simeq 4 N_{\text {coh }}$. Hence,

$$
C_{B H}\left(N_{T}\right) \frac{N_{c o h}\left(N_{T}\right)}{2 n_{*}^{2}} \operatorname{Tr}_{\text {out }}\left[\operatorname{Tr}_{\text {in }}\left[\Delta \rho_{O D}^{2}\right] \widetilde{D}\left(N_{T} ; N_{o}^{\prime}, N_{o}^{\prime \prime}\right)\left|N_{o}^{\prime}\right\rangle\left\langle N_{o}^{\prime \prime}\right|\right]=\gamma C_{B H}\left(N_{T}\right) \frac{2 N_{c o h}^{2}\left(N_{T}\right)}{n_{*}^{2}},
$$

whereby

$$
\gamma \equiv \operatorname{Tr}_{\text {out }} \operatorname{Tr}_{\text {in }}\left[\left|\Delta \rho_{O D}\right|^{2}\right]
$$

with $\gamma$ being a number of order unity.

Then, as the full trace of the density matrix should be unity, it follows that

$$
n_{*}^{2}=2 N_{c o h}^{2}\left(N_{T}\right)\left[1+\gamma C_{B H}\left(N_{T}\right)\right] .
$$

Our starting point for the next section is the reduced density matrix for the out-modes with correct normalization (up to order $C_{B H}$ ),

$$
\begin{aligned}
\rho_{S C, \text { out }}\left(N_{T} ; N_{o}^{\prime}, N_{o}^{\prime \prime}\right)= & \frac{1}{N_{c o h}\left(N_{T}\right)}\left[1-\gamma C_{B H}\left(N_{T}\right)\right] \operatorname{Tr}_{\text {in }}\left[\rho_{H}\right]\left|N_{o}^{\prime}\right\rangle\left\langle N_{o}^{\prime \prime}\right| \delta_{N_{o}^{\prime}, N_{o}^{\prime \prime}} \\
& +\frac{C_{B H}\left(N_{T}\right)}{4 N_{c o h}\left(N_{T}\right)} \operatorname{Tr}_{\text {in }}\left[\left|\Delta \rho_{O D}\right|^{2}\right] \widetilde{D}\left(N_{T} ; N_{o}^{\prime}, N_{o}^{\prime \prime}\right)\left|N_{o}^{\prime}\right\rangle\left\langle N_{o}^{\prime \prime}\right| \\
& +\frac{C_{B H}^{1 / 2}\left(N_{T}\right)}{2 N_{c o h}\left(N_{T}\right)} \operatorname{Tr}_{\text {in }}\left[\bar{\Delta}_{O D}\right] \bar{D}\left(N_{T} ; N_{o}^{\prime}, N_{o}^{\prime \prime}\right)\left|N_{o}^{\prime}\right\rangle\left\langle\left. N_{o}^{\prime \prime}\right|_{N_{o}^{\prime} \neq N_{o}^{\prime \prime}}\right.
\end{aligned}
$$




\section{$7 \quad$ In-Out entanglement}

To determine the entanglement entropy for an approximately pure state, it is appropriate to use the von Neumann entropy formula. Then the entanglement entropy per particle is ${ }^{1}$

$$
\frac{S_{\text {ent }}}{N_{\text {part }}}=-\operatorname{Tr}[\hat{\rho} \ln \hat{\rho}]
$$

where, in our case, $N_{\text {part }}=N_{\text {coh }}$.

For the Hawking part alone,

$$
\left[S_{\text {ent }}\right]_{H}=-\left[1-\gamma C_{B H}\left(N_{T}\right)\right] \operatorname{Tr}_{\text {out }} \operatorname{Tr}_{\text {in }}\left[\rho_{H} \ln \rho_{H}\right] N_{c o h}\left(N_{T}\right),
$$

where we have eliminated a factor of $\ln \left(N_{c o h}\right)$ by correcting for Gibbs' paradox for indistinguishable particles. The leading-order outcome is, of course, the expected result for a total of $N_{c o h}$ maximally entangled pairs.

\subsection{In-Out entanglement for $t<t_{\text {trans }}$}

What is left to resolve is the effect of the correction. Let us recall that we are considering a "typical" BH for $t_{c o h}<t<t_{\text {trans }}$. The extreme cases will be addressed further along.

For a matrix of the form of that in eq. (6.16), the effective perturbation parameter is $C_{B H} N_{c o h} \sim C_{B H}^{1 / 2} \ll 1[52]$. This is because the corrections of order $C_{B H}^{1 / 2}$ are strictly off-diagonal and so can only appear at quadratic order in tracing operations. Hence, we can evaluate the correction to the entanglement perturbatively.

Then, to proceed, we expand out the logarithm in the von Neumann formula to linear order in $C_{B H}$ and use the approximation $\mathcal{J} \simeq 4 N_{\text {coh }}$ (and its $\bar{D}$ analogue) as discussed above. The result (again after accounting for Gibbs' paradox) is

$$
S_{\text {ent }}=-\operatorname{Tr}_{\text {out }} \operatorname{Tr}_{\text {in }}\left[\rho_{H} \ln \rho_{H}\right] N_{c o h}\left(N_{T}\right)\left(1-\frac{(\gamma+1) C_{B H}\left(N_{T}\right)}{-\operatorname{Tr}_{\text {out }} \operatorname{Tr}_{\text {in }}\left[\rho_{H} \ln \rho_{H}\right]}\right) .
$$

One can observe that the entanglement of the in-out sector is still parametrically close to maximal, $S_{\text {ent }}\left(N_{T}\right) \sim N_{\text {coh }}\left(N_{T}\right)$.

\subsection{Qualitative analysis of the in-out entanglement for $t>t_{\text {trans }}$}

Let us now remove the constraint of typicality on the age of the BH. Early times in the evolution $\left(t<t_{c o h}\right)$ are well understood and need not concern us, but what about late in the process? From the analysis in [52], we have observed that the BH evolves in typical fashion until about one interval of coherence time before the end of evaporation. At this point, which is what we call the transparency time $t_{\text {trans }}, C_{B H}$ starts growing rapidly from a value of $C_{B H}\left(t_{\text {trans }}\right) \sim S_{B H}^{-2 / 3}(0)$ to its value of unity for a Planck-sized BH. Moreover, the information $I$ begins to rapidly emerge from the $\mathrm{BH},\left.\frac{d I}{d N_{T}}\right|_{t_{\text {trans }}} \sim 1$.

Since $C_{B H}$ is becoming large at such late times, it is evident that our previous perturbative treatment is no longer applicable. In fact, as made clear in [52], our treatments already begins to break down at the transparency time. However, by this time,

\footnotetext{
${ }^{1}$ The von Neumann formula only gives the total entanglement if the particles had first been symmetrized.
} 
at least $\left[N_{T}\right]_{\max }-N_{\text {coh }}\left(t_{\text {trans }}\right) \simeq S_{B H}(0)-N_{\text {coh }}\left(t_{\text {trans }}\right)$ particles have already been radiated away. So the number of Hawking particles which are yet to be emitted is about $N_{\text {coh }}\left(t_{\text {trans }}\right) \simeq S_{B H}^{1 / 2}\left(t_{\text {trans }}\right) \sim S_{B H}^{1 / 3}(0)$. This means that, from $t_{\text {trans }}$ until the BH evaporates completely, at most $S_{B H}^{1 / 3}(0)$ entangled pairs can be created. It is quite possible that these remaining pairs are no longer maximally entangled. However, the $\mathrm{BH}$ is now within the final stage of evaporation and there is no compelling reason to believe that the late-time horizon is cold. Any chance of forming a firewall is delayed at least until a parametrically smal 1 time before the $\mathrm{BH}$ has finally evaporated.

Let us now consider in more detailed way the evolution of the entanglement for $t>$ $t_{\text {trans }}$. As already remarked upon, we cannot make precise statements as to what transpires at times later than $t_{\text {trans }}$. In particular (and as discussed in [52]), we do not know the precise expression for the coherence scale $N_{c o h}\left(N_{T} ; N^{\prime}\right)$ in this regime.

On the other hand, contrary to our previous out-out analysis, the precise value of the coherence scale is not particularly relevant to the in-out sector at late times. This is because the number of remaining coherent pairs $N_{\text {pairs }}$ cannot be larger than the total number of Hawking particles remaining to be emitted, and so $N_{\text {pairs }}$ is no longer set by the coherence scale but rather $N_{\text {pairs }}\left(N_{T}\right)=\left[N_{T}\right]_{\max }-N_{T}$. We find this number to be smaller than the coherence scale, $N_{\text {pairs }}\left(N_{T}\right)<N_{\text {coh }}\left(N_{T} ; N^{\prime}\right)$, for $t>t_{\text {trans }}$. This follows from the qualitative estimates in [52], where $N_{c o h}\left(N_{T} ; N^{\prime}\right)$ was found to be a monotonically growing quantity after the transparency time. The only exception being $N^{\prime} \simeq N_{T} \simeq N_{\text {trans }}$; in which case, $N_{\text {coh }}$ and $N_{\text {pairs }}$ are parametrically similar.

During these late times, $C_{B H}\left(N_{T}\right)$ is becoming large and will eventually approach unity as the $\mathrm{BH}$ tends toward Planckian dimensions. As a consequence, any of the exponential suppression factors (e.g., the first one in eq. (6.8)) becomes like a theta or Heaviside function, as the numerator of the exponent $\left[C_{B H}\left(N^{\prime}\right)\left(N_{T}-N^{\prime}\right)\right]^{2}$ is a number of order unity for all choices of $N^{\prime}$ when $N_{T}$ is approaching its maximum value.

In light of the above, the reduced density matrix for the out-modes simplifies at late times,

$$
\begin{aligned}
\rho_{S C, \text { out }}\left(N_{T} ; N_{o}^{\prime}, N_{o}^{\prime \prime}\right)= & \frac{1}{N_{\text {pairs }}\left(N_{T}\right)} \operatorname{Tr}_{\text {in }}\left[\rho_{H}\right]\left|N_{o}^{\prime}\right\rangle\left\langle N_{o}^{\prime \prime}\right| \delta_{N_{o}^{\prime}, N_{o}^{\prime \prime}} \\
& +\frac{2}{N_{\text {pairs }}\left(N_{T}\right)} C_{B H}^{1 / 2}\left(N_{T}\right) \operatorname{Tr}_{\text {in }}\left[\bar{\Delta}_{O D}\right]\left|N_{o}^{\prime}\right\rangle\left\langle\left. N_{o}^{\prime \prime}\right|_{N_{o}^{\prime \prime} \neq N_{o}^{\prime}}\right. \\
& +\mathcal{O}\left[C_{B H}\right]
\end{aligned}
$$

with $C_{B H}$ now regarded as a number that is still small (relative to unity) but large enough to satisfy $C_{B H} \gg N_{c o h}^{-1}$.

The "correction" part of the matrix seems to become more important and eventually would seem dominate the diagonal part. This is because the Hawking part is diagonal with $N_{\text {pairs }}$ entries, whereas the correction is a nearly uniform matrix with $N_{\text {pairs }}^{2}-N_{\text {pairs }} \gg$ $N_{\text {pairs }}$ entries. However, notice that the off-diagonal part can only appear quadratically when a trace of some operator is evaluated. And so the correction it makes to the entropy or other physical quantities is actually suppressed by a power of $C_{B H}<1$ with respect to the diagonal contribution until such time as the $\mathrm{BH}$ approaches Planckian dimensions. 
Hence, we can expect the previous (early-time) calculation to remain roughly valid at least until the $\mathrm{BH}$ has shrunk past its regime of semiclassical validity. We can then conclude that, even at late times, $S_{\text {ent }} \sim N_{\text {pairs }}$ and the in-out entanglement remains parametrically close to maximal, while monotonically decreasing in time in the same way that $N_{\text {pairs }}$ does.

As already emphasized, these arguments are qualitative. It is, however, worthwhile to remember that the late-time horizon region can not necessarily be expected to be similar to the vacuum, contrary to our expectations at earlier times. Hence, our overall argument does not hinge on the exact fate of the in-out sector at these final stages.

\subsection{Summary of the evolution of entanglement entropy and released informa- tion}

Let us summarize the dependence of the in-out entanglement on time. For $t<t_{c o h}$ the entanglement entropy increases linearly with the number of emitted Hawking particles. For $t_{c o h}<t<t_{\text {trans }}$, the entanglement entropy is equal to $N_{c o h}$, which is a very slowly decreasing function of the number of emitted particles and can then be approximated by a constant. After $t_{\text {trans }}$, the entanglement entropy decreases to zero. We have argued that this decrease is linear in the number of particles that are yet to be emitted.

The following equation summarizes the different dependencies of the entanglement entropy:

$$
S_{\text {ent }}\left(N_{T}\right) \sim \begin{cases}N_{T} & 0 \leq N_{T} \leq N_{c o h} \\ N_{c o h} \sim \sqrt{N-N_{T}} & N_{c o h} \leq N_{T} \leq N-N^{2 / 3} \\ N-N_{T} & N-N^{2 / 3} \leq N_{T} \leq N .\end{cases}
$$

Here, we have denoted by $N$ the total number of Hawking particles emitted during the lifetime of the BH, $N=\left[N_{T}\right]_{\max } \simeq S_{B H}(0)$. (This $N$ should not to be confused with the argument of $N_{c o h}$.)

For comparison, we also recall how $S_{\text {ent }}$ evolves for the Page model [37, 38],

$$
S_{\text {ent }}^{\text {Page }}\left(N_{T}\right) \sim \begin{cases}N_{T} & 0 \leq N_{T} \leq N / 2 \\ N-N_{T} & N / 2 \leq N_{T} \leq N .\end{cases}
$$

Let us further recall how the released information $I$ depends on the number of emitted particles, as calculated in [52],

$$
I\left(N_{T}\right) \sim \begin{cases}N_{T} \frac{1}{\left(N-N_{T}\right)^{1 / 2}} & N_{T} \ll N-N^{2 / 3} \\ N_{T} \frac{N}{\left(N-N_{T}\right)^{3 / 2}} & N_{T} \lesssim N-N^{2 / 3}\end{cases}
$$

and

$$
\frac{d I}{d N_{T}} \sim 1 \quad N_{T} \sim N-N^{2 / 3}
$$

For comparison, the evolution of the released information for the Page model goes as

$$
I^{\text {Page }}\left(N_{T}\right) \sim \begin{cases}0 & 0 \leq N_{T} \leq N / 2 \\ 2\left(N_{T}-N / 2\right) & N / 2 \leq N_{T} \leq N\end{cases}
$$




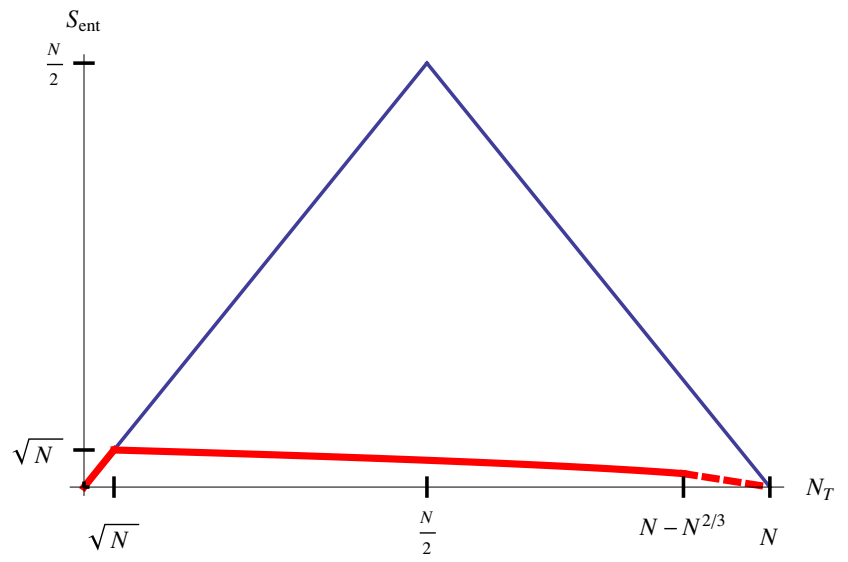

Figure 1. Entanglement entropy $S_{\text {ent }}$ of the Hawking pairs, as a function of the number of emitted Hawking particles $N_{T}$. This is shown both for the Page model (thin, blue) and ours (thick, red). The units are arbitrary. The main difference is the maximal value of the entanglement entropy, which scales as $N_{T}$ for the Page model and as $\sqrt{N_{T}}$ for ours. The decrease in $S_{\text {ent }}$ starts when $d I / d N_{T} \sim 1$.

The dependence of $S_{\text {ent }}$ on the number of emitted particles is shown in figure 1.

It should be emphasized that we are only considering the entanglement between Hawking modes and their negative-energy partners and not the entanglement between the Hawking modes and the rest of the interior of the $\mathrm{BH}$. This is the reason that the graph in figure 1 takes the flattened form that it does. This distinction between partners and the interior of the $\mathrm{BH}$ is of no consequence to Page because of his indifference to the horizon. It does, however, make a difference for us because our framework is such that it limits the number of negative-energy partners at any given time to $N_{c o h} \sim S_{B H}^{1 / 2}$; which necessarily limits the amount of entanglement in the same way. Physically, the semiclassical horizon is acting to shield all but a fraction $N_{c o h}$ of the matter modes.

On the other hand, the breakdown of the semiclassical picture at late times (see subsection 1.1 for a discussion) suggests that all the information can still be released once the (quantum) horizon is no longer acting as a causal barrier. Some qualtitative estimates in [52] along with eq. (7.8) are in support of this argument. We do, however, expect to put this claim on a more rigorous level at a later time [53].

The most significant physical difference between our model and the Page model is the incorporation (or not) of a horizon. Page basically disregards the presence of a horizon and treats the $\mathrm{BH}$ evaporation in purely information-theoretic terms. He assumes that the offdiagonal elements of the density matrix are distributed randomly, with strength controlled by the dimensionality of the entire $\mathrm{BH}$ Hilbert space. On the other hand, our framework is based on the presence of a semiclassical horizon. Had we treated the horizon classically, there would be no off-diagonal elements as is the case in Hawking's calculation. But, because our horizon is semiclassical, not all the off-diagonal elements are vanishing. The number of non-vanishing off-diagonal elements is controlled by the wavefunction of the BH; specifically, the width of the Gaussian. The density matrix and its physical consequences 
then follows. This leads us to a density matrix with many zeroes, which would be viewed by Page as a very atypical matrix.

Because Page essentially ignores the horizon, the distinction between partners and interior matter is of no consequence to his model. It does, however, make a difference for us because our framework is such that it limits the number of negative-energy partners at any given time to $N_{c o h} \sim S_{B H}^{1 / 2}$, which necessarily limits the amount of entanglement in the same way. This upper bound on the entanglement explains the flattening of the red curve in figure 1. Physically, the semiclassical horizon is acting to shield all but a fraction $N_{c o h}$ of the matter modes. This being a consequence of our choice of $\mathrm{BH}$ wavefunction, which determines the transparency of the horizon.

\section{Discussion}

We have shown that the in-out sector of the $\mathrm{BH}$ radiation is close to maximally entangled; at least until the transparency time, when our perturbative analysis begins to break down. Additionally, the entanglement between in- and out-modes is limited to a maximal value of $N_{c o h} \simeq \sqrt{S_{B H}}$ that is parametrically smaller than the total number of emitted Hawking particles. This limitation can be attributed to the regular recycling of partnered modes over a time scale that is set by the quantum width of the wavefunction for the evaporating BH.

The limited dimensionality of the boundary region between the interior and exterior Hilbert spaces is central. This restriction can be attributed to incorporating both the wavefunction for the $\mathrm{BH}$ geometry and the back-reaction on the $\mathrm{BH}$ due to the emitted particles. When $t<t_{\text {trans }}$, the semiclassical corrections to the in-out density matrix are insignificant - the Hawking pairs are highly entangled with or without them. On the other hand, these corrections enable information to be transferred to the outgoing radiation via the off-diagonal corrections to the Hawking matrix. Their presence follows from treating the $\mathrm{BH}$ consistently as a quantum object.

Let us recall [52], where qualitative considerations were used to conclude that the outgoing radiation starts to purify at the same late time when the entanglement entropy is beginning to decrease. The implication for our framework is that, for times earlier than the transparency time, any duplication of entanglement or purity $[32,33]$ is avoided without the need to modify the rules of quantum mechanics. Hence, our framework is immune against the formation of firewalls and horizons are cold, at least until one coherence time before the end of evaporation.

Let us reconsider the Page model and its associated firewall. The implicit assumption in this model is that the number of strongly entangled pairs becomes of order $S_{B H}$ by the Page time [37, 38]. But this is also supposed to be the time when information begins to rapidly emerge from the $\mathrm{BH}$, and one encounters the inevitable conflict of interests. The transfer of information means the transfer of entanglement from the partnered pairs to the late-early radiation. One is then faced with the prospect of a firewall or, otherwise, a means for circumventing the rule about monogamy of entanglement. As just mentioned, in our model of $\mathrm{BH}$ evaporation, this issue is postponed until a much later time, when the $\mathrm{BH}$ stops being semiclassical. In our model, the interior system that purifies the outgoing 
radiation has an additional component, the $\mathrm{BH}$ wavefunction. This additional component is likely representing the collapsed matter from an in-falling observer's perspective.

Furthermore, even for our model, the in-out entanglement does stray from its maximal value by small amounts. This result suggests the interesting possibility that some part of the firewall idea still survives. Very old BHs do seem to have different properties than younger ones. The question then arises: How much of a deviation is needed before a firewall forms? A related question is how strongly did our conclusions depend on the precise choice for the wavefunction and on our model of back-reaction. We hope to make these questions more precise and provide quantitative answers in a future article [55].

\section{Acknowledgments}

We thank Sunny Itzhaki for discussions and useful comments on the manuscript. The research of RB was supported by the Israel Science Foundation grant no. 239/10. The research of AJMM received support from an NRF Incentive Funding Grant 85353 and an NRF KIC Grant 83407. AJMM thanks Ben Gurion University for their hospitality during his visit.

Open Access. This article is distributed under the terms of the Creative Commons Attribution License (CC-BY 4.0), which permits any use, distribution and reproduction in any medium, provided the original author(s) and source are credited.

\section{References}

[1] S.W. Hawking, Black hole explosions, Nature 248 (1974) 30 [INSPIRE].

[2] S.W. Hawking, Particle Creation by Black Holes, Commun. Math. Phys. 43 (1975) 199 [Erratum ibid. 46 (1976) 206-206] [INSPIRE].

[3] S.W. Hawking, Breakdown of Predictability in Gravitational Collapse, Phys. Rev. D 14 (1976) 2460 [inSPIRE].

[4] D.N. Page, Black hole information, hep-th/9305040 [INSPIRE].

[5] S.B. Giddings, Comments on information loss and remnants, Phys. Rev. D 49 (1994) 4078 [hep-th/9310101] [INSPIRE].

[6] S.D. Mathur, What Exactly is the Information Paradox?, Lect. Notes Phys. 769 (2009) 3 [arXiv:0803.2030] [INSPIRE].

[7] S.D. Mathur, The Information paradox: A Pedagogical introduction, Class. Quant. Grav. 26 (2009) 224001 [arXiv:0909.1038] [InSPIRE].

[8] S.D. Mathur, What the information paradox is not, arXiv:1108.0302 [INSPIRE].

[9] A. Almheiri, D. Marolf, J. Polchinski and J. Sully, Black Holes: Complementarity or Firewalls?, JHEP 02 (2013) 062 [arXiv:1207.3123] [INSPIRE].

[10] N. Itzhaki, Is the black hole complementarity principle really necessary?, hep-th/9607028 [INSPIRE]. 
[11] S.L. Braunstein, S. Pirandola and K. Życzkowski, Better Late than Never: Information Retrieval from Black Holes, Phys. Rev. Lett. 110 (2013) 101301 [arXiv:0907.1190] [INSPIRE].

[12] S.D. Mathur and C.J. Plumberg, Correlations in Hawking radiation and the infall problem, JHEP 09 (2011) 093 [arXiv:1101.4899] [INSPIRE].

[13] L. Susskind, Singularities, Firewalls and Complementarity, arXiv:1208.3445 [INSPIRE].

[14] L. Susskind, The Transfer of Entanglement: The Case for Firewalls, arXiv:1210.2098 [INSPIRE].

[15] R. Bousso, Complementarity Is Not Enough, Phys. Rev. D 87 (2013) 124023 [arXiv: 1207.5192] [INSPIRE].

[16] Y. Nomura, J. Varela and S.J. Weinberg, Complementarity Endures: No Firewall for an Infalling Observer, JHEP 03 (2013) 059 [arXiv:1207.6626] [INSPIRE].

[17] Y. Nomura, J. Varela and S.J. Weinberg, Black Holes, Information and Hilbert Space for Quantum Gravity, Phys. Rev. D 87 (2013) 084050 [arXiv:1210.6348] [INSPIRE].

[18] S.D. Mathur and D. Turton, Comments on black holes I: The possibility of complementarity, JHEP 01 (2014) 034 [arXiv: 1208.2005] [INSPIRE].

[19] A. Giveon and N. Itzhaki, String Theory Versus Black Hole Complementarity, JHEP 12 (2012) 094 [arXiv:1208.3930] [INSPIRE].

[20] S.G. Avery, B.D. Chowdhury and A. Puhm, Unitarity and fuzzball complementarity: 'Alice fuzzes but may not even know it!', JHEP 09 (2013) 012 [arXiv:1210.6996] [INSPIRE].

[21] K. Larjo, D.A. Lowe and L. Thorlacius, Black holes without firewalls, Phys. Rev. D 87 (2013) 104018 [arXiv:1211.4620] [INSPIRE].

[22] E. Verlinde and H. Verlinde, Black Hole Entanglement and Quantum Error Correction, JHEP 10 (2013) 107 [arXiv:1211.6913] [INSPIRE].

[23] E. Verlinde and H. Verlinde, Passing through the Firewall, arXiv:1306.0515 [INSPIRE].

[24] E. Verlinde and H. Verlinde, Black Hole Information as Topological Qubits, arXiv: 1306.0516 [INSPIRE].

[25] K. Papadodimas and S. Raju, An Infalling Observer in AdS/CFT, JHEP 10 (2013) 212 [arXiv: 1211.6767] [INSPIRE].

[26] A. Almheiri, D. Marolf, J. Polchinski, D. Stanford and J. Sully, An Apologia for Firewalls, JHEP 09 (2013) 018 [arXiv:1304.6483] [INSPIRE].

[27] D.A. Lowe and L. Thorlacius, Pure states and black hole complementarity, Phys. Rev. D 88 (2013) 044012 [arXiv: 1305.7459] [INSPIRE].

[28] J. Maldacena and L. Susskind, Cool horizons for entangled black holes, Fortsch. Phys. 61 (2013) 781 [arXiv:1306.0533] [INSPIRE].

[29] D.N. Page, Excluding Black Hole Firewalls with Extreme Cosmic Censorship, arXiv:1306.0562 [INSPIRE].

[30] M. Van Raamsdonk, Evaporating Firewalls, arXiv:1307.1796 [INSPIRE].

[31] D. Marolf and J. Polchinski, Gauge/Gravity Duality and the Black Hole Interior, Phys. Rev. Lett. 111 (2013) 171301 [arXiv:1307.4706] [INSPIRE]. 
[32] R. Bousso, Firewalls From Double Purity, Phys. Rev. D 88 (2013) 084035 [arXiv: 1308.2665] [INSPIRE].

[33] R. Bousso, Frozen Vacuum, Phys. Rev. Lett. 112 (2014) 041102 [arXiv:1308.3697] [INSPIRE].

[34] K. Papadodimas and S. Raju, State-Dependent Bulk-Boundary Maps and Black Hole Complementarity, Phys. Rev. D 89 (2014) 086010 [arXiv:1310.6335] [InSPIRE].

[35] E. Verlinde and H. Verlinde, Behind the Horizon in AdS/CFT, arXiv:1311.1137 [INSPIRE].

[36] S.L. Braunstein and S. Pirandola, Evaporating black holes have leaky horizons or exotic atmospheres, arXiv:1311.1326 [INSPIRE].

[37] D.N. Page, Average entropy of a subsystem, Phys. Rev. Lett. 71 (1993) 1291 [gr-qc/9305007] [INSPIRE].

[38] D.N. Page, Information in black hole radiation, Phys. Rev. Lett. 71 (1993) 3743 [hep-th/9306083] [INSPIRE].

[39] L. Susskind, L. Thorlacius and J. Uglum, The Stretched horizon and black hole complementarity, Phys. Rev. D 48 (1993) 3743 [hep-th/9306069] [INSPIRE].

[40] C.R. Stephens, G. 't Hooft and B.F. Whiting, Black hole evaporation without information loss, Class. Quant. Grav. 11 (1994) 621 [gr-qc/9310006] [INSPIRE].

[41] R. Brustein, Origin of the blackhole information paradox, Fortsch. Phys. 62 (2014) 255 [arXiv:1209.2686] [INSPIRE].

[42] G. Dvali and C. Gomez, Black Hole's Quantum N-Portrait, Fortsch. Phys. 61 (2013) 742 [arXiv:1112.3359] [INSPIRE].

[43] G. Dvali and C. Gomez, Black Hole's 1/N Hair, Phys. Lett. B 719 (2013) 419 [arXiv: 1203.6575] [INSPIRE].

[44] G. Dvali and C. Gomez, Black Holes as Critical Point of Quantum Phase Transition, Eur. Phys. J. C 74 (2014) 2752 [arXiv: 1207.4059] [INSPIRE].

[45] G. Dvali and C. Gomez, Black Hole Macro-Quantumness, arXiv:1212.0765 [INSPIRE].

[46] G. Dvali, C. Gomez and D. Lüst, Black Hole Quantum Mechanics in the Presence of Species, Fortsch. Phys. 61 (2013) 768 [arXiv:1206.2365] [INSPIRE].

[47] G. Dvali, D. Flassig, C. Gomez, A. Pritzel and N. Wintergerst, Scrambling in the Black Hole Portrait, Phys. Rev. D 88 (2013) 124041 [arXiv:1307.3458] [InSPIRE].

[48] R. Brustein and M. Hadad, Wave function of the quantum black hole, Phys. Lett. B 718 (2012) 653 [arXiv:1202.5273] [INSPIRE].

[49] R. Brustein and J. Kupferman, Black hole entropy divergence and the uncertainty principle, Phys. Rev. D 83 (2011) 124014 [arXiv: 1010.4157] [InSPIRE].

[50] R. Brustein and A.J.M. Medved, Semiclassical black holes expose forbidden charges and censor divergent densities, JHEP 09 (2013) 108 [arXiv: 1302.6086] [INSPIRE].

[51] R. Brustein and A.J.M. Medved, Restoring predictability in semiclassical gravitational collapse, JHEP 09 (2013) 015 [arXiv:1305.3139] [INSPIRE].

[52] R. Brustein and A.J.M. Medved, Phases of information release during black hole evaporation, JHEP 02 (2014) 116 [arXiv:1310.5861] [INSPIRE]. 
[53] R. Brustein and A.J.M. Medved, How black holes burn, to appear.

[54] P. Hayden and J. Preskill, Black holes as mirrors: Quantum information in random subsystems, JHEP 09 (2007) 120 [arXiv:0708.4025] [INSPIRE].

[55] R. Brustein and A.J.M. Medved, Firewalls, smoke and mirrors, arXiv:1401.1401 [INSPIRE]. 